ARTICLE

https://doi.org/10.1038/s41467-019-10495-4

\title{
ABCB1 protects bat cells from DNA damage induced by genotoxic compounds
}

Javier Koh', Yoko Itahana (10 ${ }^{1}$, Ian H. Mendenhall ${ }^{2}$, Dolyce Low (D) ${ }^{2}$, Eunice Xin Yi Soh ${ }^{3}$, Alvin Kunyao Guo, Yok Teng Chionh², Lin-Fa Wang $\mathbb{1}^{2}$ \& Koji Itahana (i) ${ }^{1}$

Bats are unusual mammals, with the ability to fly, and long lifespans. In addition, bats have a low incidence of cancer, but the mechanisms underlying this phenomenon remain elusive. Here we discovered that bat cells are more resistant than human and mouse cells to DNA damage induced by genotoxic drugs. We found that bat cells accumulate less chemical than human and mouse cells, and efficient drug efflux mediated by the $A B C$ transporter $A B C B 1$ underlies this improved response to genotoxic reagents. Inhibition of $A B C B 1$ triggers an accumulation of doxorubicin, DNA damage, and cell death. ABCB1 is expressed at higher levels in several cell lines and tissues derived from bats compared to humans. Furthermore, increased drug efflux and high expression of $A B C B 1$ are conserved across multiple bat species. Our findings suggest that enhanced efflux protects bat cells from DNA damage induced by genotoxic compounds, which may contribute to their low cancer incidence.

\footnotetext{
${ }^{1}$ Programme in Cancer \& Stem Cell Biology, Duke-NUS Medical School, 8 College Road, Singapore 169857, Singapore. ${ }^{2}$ Programme in Emerging Infectious Diseases, Duke-NUS Medical School, 8 College Road, Singapore 169857, Singapore. ${ }^{3}$ School of Applied Science, Temasek Polytechnic, 21 Tampines Avenue 1, Singapore 529757, Singapore. Correspondence and requests for materials should be addressed to L.F.W. (email: linfa.wang@duke-nus.edu.sg) or to K.I. (email: koji.itahana@duke-nus.edu.sg)
} 
C ancer is a leading cause of death among aging populations. Current cancer treatments are inefficient and have unacceptable side-effects, due in part to the biological complexity and incomplete understanding of the disease ${ }^{1}$. Therefore, there is an urgent need to develop alternative therapeutics. Indepth understanding of tumour suppressive mechanisms can provide effective strategies to treat and prevent cancer.

Most cancer research utilises cancer-prone, short-lived animals such as mouse and rat, which are amenable to genetic manipulation. Although these model organisms share some tumour suppressor mechanisms with humans, emerging evidence suggests that long-lived mammals have evolved unique and robust anti-cancer strategies ${ }^{2}$. For example, genome and transcriptome analyses of the bowhead whale, a large and long-lived mammal with a low rate of cancer, revealed a positive evolutionary selection of the excision repair cross-complementation group 1 (ERCC1) gene and duplication of the proliferating cell nuclear antigen (PCNA) gene, both DNA repair genes ${ }^{3}$. These data imply that an enhanced DNA repair pathway might protect whales from cancer by lowering the mutation frequency. Genomic analysis of elephants, which also have a low cancer incidence, uncovered a high number of TP53 pseudogenes, and elephant cells displayed an enhanced TP53-dependent DNA damage response compared to human cells ${ }^{4,5}$.

Some small mammals also show remarkable cancer resistance. Early contact inhibition is a unique mechanism of tumour suppression in the naked mole rat, mediated by the secretion of highmolecular-mass hyaluronic acid ${ }^{6,7}$. Blind mole rats also exhibit remarkable cancer resistance by inducing concerted necrotic cell death in response to hyperplasia ${ }^{8}$ and by having a stronger extracellular matrix to restrict tumour growth and metastasis 9 . Unravelling the mechanisms underlying low cancer rates provides important perspectives and insights into cancer biology and potential treatment strategies for humans.

Bats are small, long-lived mammals with an extremely low incidence of cancer ${ }^{2,10}$. They are the second largest order of mammals in the world ${ }^{11}$, the only mammal capable of poweredwing flight, and an asymptomatic reservoir for many deadly viruses ${ }^{10}$. Their longevity data predominately come from fieldbased studies, and therefore, their true longevities may be underestimated, and they may live longer than these reported records ${ }^{12-14}$. In general, longevity is positively correlated with the body size ${ }^{12,13}$. Austad and Fischer ${ }^{13}$ defined the longevity quotient (LQ) which takes the consideration of body mass in the estimated maximum lifespan of individual mammalian species. Bats possess one of the highest LQ value among the mammal order ${ }^{12,13}$, indicating that bats live much longer than other mammals of equivalent size. Their higher LQ makes bats interesting species to study since they may have unique tumour suppressive mechanisms compared to humans. Only a handful of cases of tumours have been recorded to date for bats in captivity ${ }^{15-17}$. However, the underlying mechanisms of tumour suppression in bats are still not fully understood. To understand such mechanisms, we previously performed genomic analyses of Pteropus alecto (P. alecto) and Myotis davidii, and discovered that the DNA damage response (DDR) pathway of bats had undergone positive evolutionary selection ${ }^{18}$. The DDR pathway is the major mechanism that detects and repairs damaged DNA and highly conserved in a diverse range of organisms ${ }^{19}$. DNA damage can induce cancer via multiple mechanisms, including genomic instability, inactivation of tumour suppressors or activation of oncogenes ${ }^{20}$. Living organisms are constantly exposed to environmental xenobiotics and internal metabolic by-products, some of which can possess genotoxic properties that can lead to DNA damage $^{21,22}$. Elucidating whether and how bats efficiently resist DNA damage will advance our understanding of cancer biology.
Here, we describe a role for the ATP-binding cassette (ABC) transporter $\mathrm{ABCB} 1$ in DNA damage resistance in bats. We propose genotoxic efflux as a potential tumour suppressive mechanism that contributes to the low incidence of cancer in bats.

\section{Results}

Bat-derived cells respond distinctively to chemical exposure. To explore DNA damage resistant mechanisms in bat, we first examined the DNA damage response in bat, human and mouse cells exposed to different DNA damaging treatments.

First, we exposed PaLung (normal lung fibroblast from $P$. alecto), WI-38 (human normal lung fibroblast) and MEF (mouse embryonic fibroblast) cells to $\gamma$-irradiation, which induces double-strand breaks (DSBs) in DNA via free radical formation ${ }^{23}$. We exposed cells to $10 \mathrm{~Gy}$ of $\gamma$-irradiation and monitored the well-established DNA DSB marker phosphorylated histone 2A variant $\mathrm{X}(\gamma \mathrm{H} 2 \mathrm{AX})^{24}$. We found that $\gamma \mathrm{H} 2 \mathrm{AX}$ accumulated and diminished with similar kinetics in PaLung, WI-38 and MEF cells exposed to $\gamma$-irradiation (Fig. 1a). P. alecto PaKiT03 cells (kidney cells transformed with SV40 large $\mathrm{T}$ antigen) and human HEK293T cells (embryonic kidney cell transformed with SV40 large $\mathrm{T}$ antigen) also showed similar changes in $\gamma \mathrm{H} 2 \mathrm{AX}$ levels in response to $\gamma$-irradiation (Supplementary Fig. 1A).

To verify that elevated $\gamma \mathrm{H} 2 \mathrm{AX}$ represented a DSB response rather than a preapoptotic signal associated with high levels of pan-nuclear $\gamma \mathrm{H} 2 \mathrm{AX}^{25}$, we monitored $\gamma \mathrm{H} 2 \mathrm{AX}$ in cells by immunofluorescence. We found that cells exposed to $\gamma$ irradiation displayed $\gamma \mathrm{H} 2 \mathrm{AX}$ foci rather than pan-nuclear accumulation (Supplementary Fig. 1B), consistent with DSBs. To further confirm these results, we detected the DNA repair protein p53-binding protein 1 (53BP1) by immunofluorescence. $53 \mathrm{BP} 1$ is recruited to DSBs to facilitate non-homologous endjoining repair and is released upon repair ${ }^{26,27}$. We observed an increase in the number of 53BP1 foci in PaLung and WI-38 cells exposed to $\gamma$-irradiation, which reduced gradually and similarly over time (Fig. 1b). On the other hand, the number of 53BP1 foci increased slowly in $\gamma$-irradiated MEFs and remained high at least $12 \mathrm{~h}$ after irradiation (Fig. 1b), consistent with a previous report about DNA repair ${ }^{28}$. Together, these results suggest that lung fibroblasts from $P$. alecto and human are similarly sensitive and responsive to DNA damage induced by ionising radiation, whereas MEFs display a slightly slower response to the same treatment.

Next, we treated the same set of cell lines with the chemotherapeutic drug etoposide $(50 \mu \mathrm{M})$. Etoposide inhibits topoisomerase $\mathrm{II}^{29}$ and thus induces DNA DSBs. We treated cells for $3 \mathrm{~h}$, washed away the drug, and monitored the levels of $\gamma \mathrm{H} 2 \mathrm{AX}$ over time after drug removal. $\gamma \mathrm{H} 2 \mathrm{AX}$ was similarly induced by etoposide in all three cell lines (Fig. 1c, at $0 \mathrm{~h}$ time point after treatment). Unexpectedly, $\gamma \mathrm{H} 2 \mathrm{AX}$ levels returned to almost basal levels within 1-3 h of etoposide removal in PaLung cells, whereas it remained elevated for at least $12 \mathrm{~h}$ in WI-38 and MEF cells (Fig. 1c). Similarly, $\gamma \mathrm{H} 2 \mathrm{AX}$ levels returned to basal levels within $3 \mathrm{~h}$ of etoposide removal in P. alecto PaKiT03 cells, but remained high in human HEK293T cells (Supplementary Fig. 1C). These results suggest that $P$. alecto cells are more resistant than human and mouse cells to etoposide-induced DNA damage.

Bat cells accumulate less doxorubicin than human and mouse cells. To extend our observations, we evaluated the response of these cells to the chemotherapy reagent doxorubicin. Like etoposide, doxorubicin inhibits topoisomerase II and induces DNA DSBs that lead to $\gamma \mathrm{H} 2 \mathrm{AX}$ induction. However, high 
a
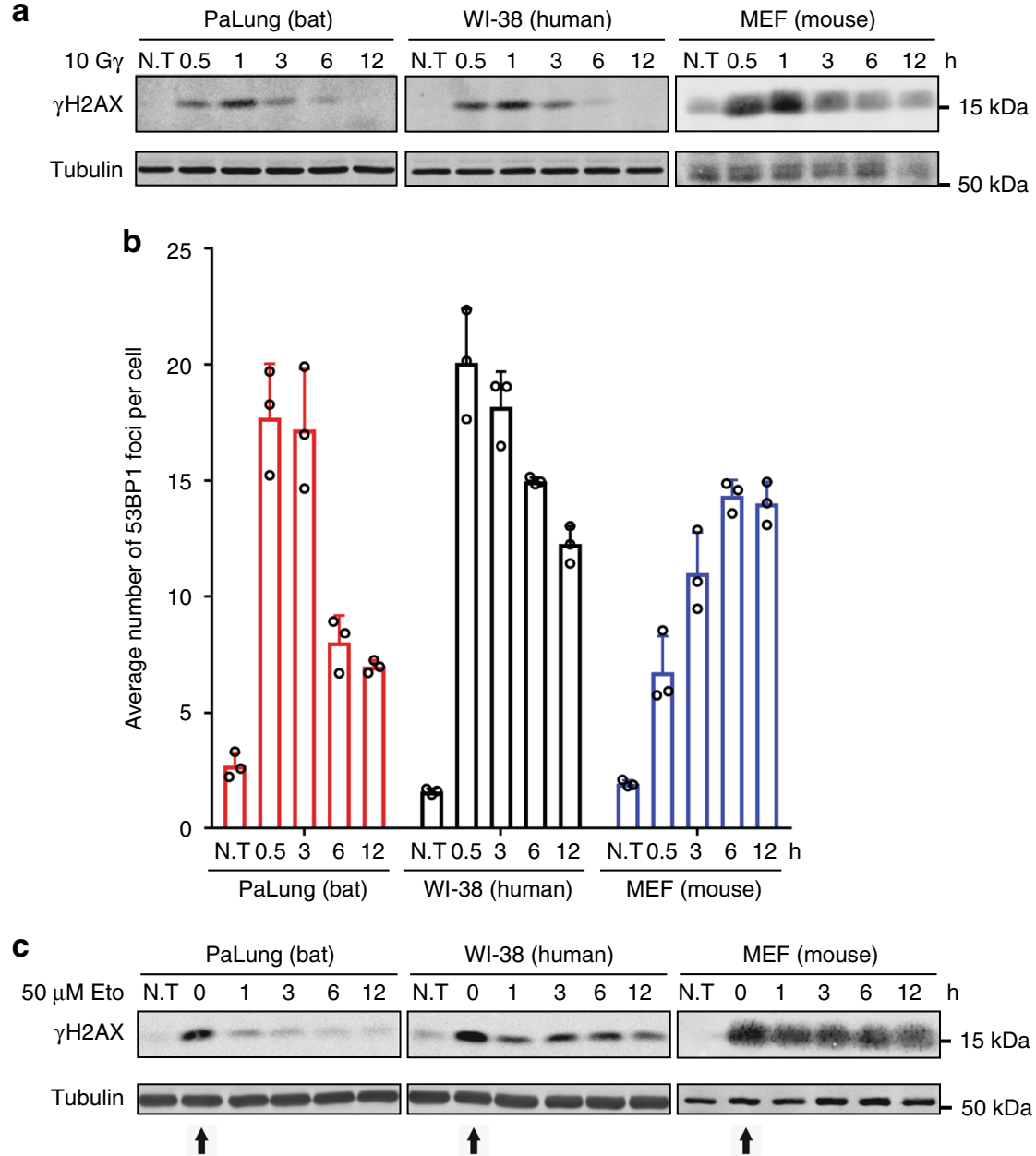

Fig. $1 \gamma \mathrm{H} 2 \mathrm{AX}$ and 53BP1 responses to $\gamma$-irradiation and etoposide in bat, human and mouse cells. a Western blot analysis of $\gamma \mathrm{H} 2 \mathrm{AX}$ in PaLung, WI-38 and MEF cells exposed to $10 \mathrm{~Gy}$ of $\gamma$-irradiation. Protein lysates were harvested at the indicated time points. Tubulin was used as a loading control. $\mathbf{b}$ Analysis of the average number of 53BP1 foci per cell for PaLung, WI-38 and MEF cells treated with $10 \mathrm{~Gy}$ of $\gamma$-irradiation. Immunofluorescence staining of 53BP1 was performed at the indicated time points. The number of foci in a minimum of 100 cells was quantified. Bars represent the means \pm SDs of three independent experiments. c Western blot analysis of $\gamma \mathrm{H} 2 \mathrm{AX}$ in PaLung, WI-38 and MEF cells treated with $50 \mu \mathrm{M}$ etoposide (Eto) for $3 \mathrm{~h}$, followed by drug-free medium up to $12 \mathrm{~h}$ (starting at $t=0 \mathrm{~h}$, indicated by arrow). Protein lysates were harvested at the indicated time points. Tubulin was used as a loading control. N.T stands for no treatment. All Western blot results shown are representative of at least three experimental repeats

concentrations of doxorubicin can cause histone eviction that leads to reduced levels of $\gamma \mathrm{H} 2 \mathrm{AX}$ in human cells ${ }^{30}$. Indeed, WI38 cells treated for $3 \mathrm{~h}$ with increasing concentrations of doxorubicin showed a dose-dependent decrease in $\gamma \mathrm{H} 2 \mathrm{AX}$ levels (Fig. 2a), consistent with histone eviction ${ }^{30}$. MEFs displayed a similar dose-dependent decrease in $\gamma \mathrm{H} 2 \mathrm{AX}$ levels (Fig. 2a). On the other hand, PaLung cells displayed a dose-dependent increase in $\gamma \mathrm{H} 2 \mathrm{AX}$ levels (Fig. 2a), consistent with increased DNA damage but without histone eviction.

As we did with etoposide, we treated cells with $5 \mu \mathrm{M}$ doxorubicin for $3 \mathrm{~h}$, washed away the drug, and monitored $\gamma \mathrm{H} 2 \mathrm{AX}$ levels over time for up to $12 \mathrm{~h}$. We found that $\gamma \mathrm{H} 2 \mathrm{AX}$ in PaLung cells started to decrease immediately after washout and returned to almost basal levels within $6 \mathrm{~h}$ (Fig. 2b). In contrast, $\gamma \mathrm{H} 2 \mathrm{AX}$ levels in WI-38 and MEF cells accumulated after washout and were sustained at high levels until the last time point we monitored (Fig. 2b). The accumulation of $\gamma \mathrm{H} 2 \mathrm{AX}$ after doxorubicin washout was similarly observed in various human cell lines, but not in various $P$. alecto cell lines (Supplementary Fig. 2A).

To monitor DSB formation, we again detected $\gamma \mathrm{H} 2 \mathrm{AX}$ by immunofluorescence. We found that doxorubicin-treated
PaLung, WI-38, and MEF cells displayed $\gamma \mathrm{H} 2 \mathrm{AX}$ foci but not pan-nuclear staining, consistent with DSBs (Supplementary Fig. 2B). Similar to $\gamma \mathrm{H} 2 \mathrm{AX}$ levels, 53BP1 foci in PaLung cells diminished soon after removal of $5 \mu \mathrm{M}$ of doxorubicin (Fig. 2c). In contrast, 53BP1 foci accumulated in WI-38 and MEF cells after removal of doxorubicin (Fig. 2c).

Together, these findings suggest that treatment with $5 \mu \mathrm{M}$ doxorubicin does not lead to histone eviction in P. alecto cells. In contrast, similarly treated human and mouse cells display transient histone eviction; the accumulation of $\gamma \mathrm{H} 2 \mathrm{AX}$ in human and mouse cells over time might reflect a decrease in intracellular doxorubicin levels, such that there is DNA damage without histone eviction.

We hypothesised that $P$. alecto cells accumulate less doxorubicin than similarly treated human and mouse cells, which would protect them from histone eviction since the only high dose of doxorubicin is known to cause histone eviction ${ }^{30}$ (Fig. 2a). To test this hypothesis, we treated the cells with increasing doses of doxorubicin and measured intracellular doxorubicin accumulation by flow cytometry ${ }^{31}$. The amount of intracellular doxorubicin was normalised to the cellular volume since there was some variation of the cell size among the cell lines (Fig. 2d). 
a

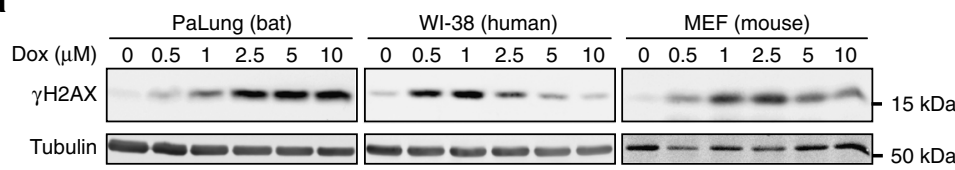

b

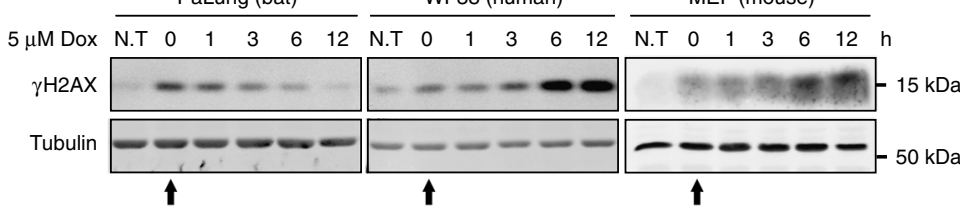

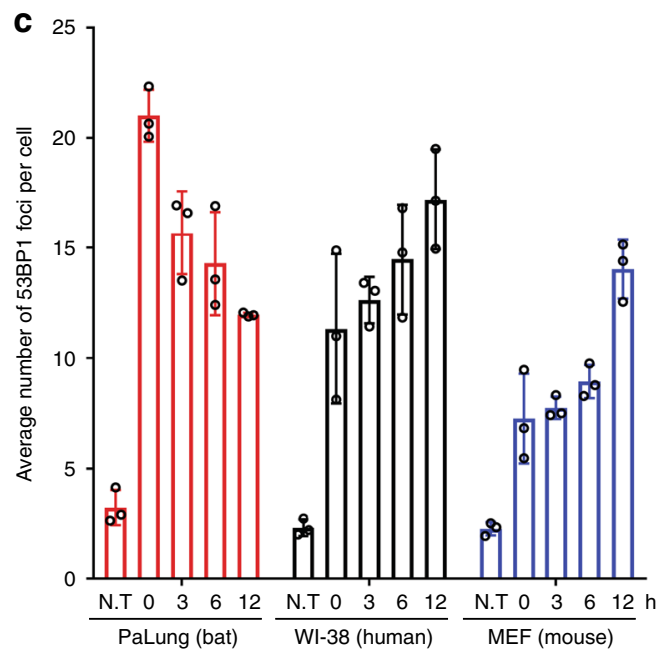

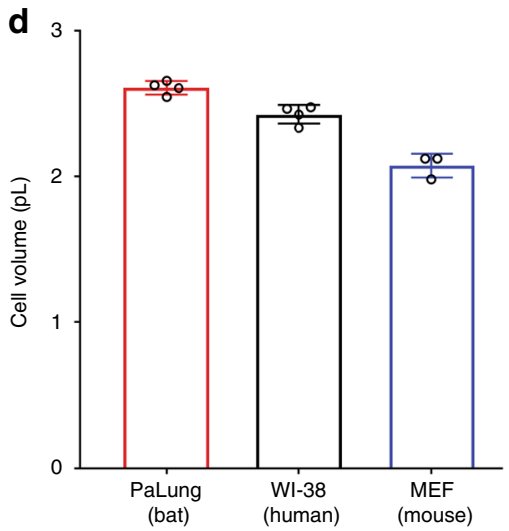

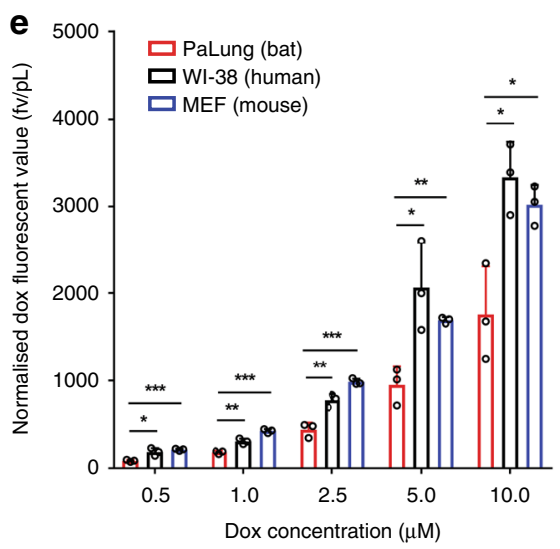

Fig. 2 Analysis of $\gamma \mathrm{H} 2 \mathrm{AX}, 53 \mathrm{BP} 1$, and intracellular accumulation of doxorubicin in bat, human, and mouse cells. a Dose titration of doxorubicin (Dox) in PaLung, WI-38 and MEF cells. Cells were treated with the indicated doses of doxorubicin for $3 \mathrm{~h}$, and protein lysates were analysed by Western blotting. Tubulin was used as a loading control. b Western blot analysis of $\gamma \mathrm{H} 2 \mathrm{AX}$ in PaLung, WI-38 and MEF cells treated with $5 \mu \mathrm{M}$ Dox for $3 \mathrm{~h}$, followed by drugfree medium up to $12 \mathrm{~h}$ (starting at $t=0 \mathrm{~h}$, indicated by arrow). Protein lysates were harvested at the indicated time points. Tubulin was used as a loading control. N.T stands for no treatment. c Analysis of the average number of 53BP1 foci per cell for PaLung, WI-38 and MEF cells. Cells were treated with $5 \mu \mathrm{M}$ Dox for $3 \mathrm{~h}$, followed by drug-free medium up to $12 \mathrm{~h}$. Immunofluorescence staining of 53BP1 was performed at the indicated time points. The number of foci in a minimum of 100 cells was quantified. N.T stands for no treatment. $\mathbf{d}$ The volume of the indicated cell lines. e Doxorubicin accumulation in PaLung, WI-38 and MEF cells after $3 \mathrm{~h}$ of incubation at the indicated concentration. The amount of accumulated doxorubicin in cells was analysed by flow cytometry. The mean fluorescence value ( $\mathrm{fv}$ ) of doxorubicin is normalised to cell volume ( $\mathrm{pL}$ ). $\mathbf{f}$ Time course of doxorubicin accumulation in PaLung, WI38 , and MEF cells. Cells were treated with $5 \mu \mathrm{M}$ doxorubicin for the indicated time and analysed by flow cytometry. The mean fluorescence value of doxorubicin accumulated in each cell line is normalised to cell volume. All Western blot results shown are representative of at least three experimental repeats. Bars represent the means \pm SDs of at least three independent experiments. Statistical significances were calculated using unpaired student's twosided $t$ test. $p<0.05$ is represented with ${ }^{*}, p<0.01$ with ${ }^{* *}$ and $p<0.001$ with ***

We found that the amount of doxorubicin within PaLung cells was significantly lower than that within WI-38 and MEF cells, at all the treatment concentrations (Fig. 2e) and times (Fig. 2f). Similar trends were observed in $P$. alecto PaKiT03 cells in comparison with human HEK293T cells (Supplementary Fig. 2CE). Together, these results suggest that $P$. alecto cells are more resistant than human and mouse cells to doxorubicin-induced DNA damage at least in part due to reduced accumulation of doxorubicin.

Bat cells exhibit efficient drug efflux via $A B C$ transporters. Intracellular drug concentrations are influenced by the balance of drug influx and efflux. $\mathrm{ABC}$ transporters are the primary transporters that efflux genotoxic substances, thereby reducing intracellular xenobiotic concentrations ${ }^{32}$. To determine if $\mathrm{ABC}$ transporters contribute to the difference in doxorubicin accumulation between bat, human and mouse cells, we used verapamil (Vera) to inhibit efflux via $\mathrm{ABC}$ transporters ${ }^{32}$, and assessed doxorubicin accumulation by fluorescence microscopy and flow cytometry. Specifically, we pre-treated cells with or without verapamil for $30 \mathrm{~min}$, added doxorubicin for $3 \mathrm{~h}$ and measured intracellular doxorubicin levels. No notable differences were observed in the doxorubicin fluorescence intensities between control and verapamil-pre-treated WI-38 cells (Fig. 3a, b), suggesting that WI-38 cells exhibit no endogenous drug efflux capability via ABC transporters. Similarly, no obvious differences were detected in doxorubicin accumulation between control and verapamil-pre-treated MEF cells (Fig. 3a, b) and HEK293T cells (Fig. $3 \mathrm{~b}$ and Supplementary Fig. 3). In contrast, verapamil-pretreatment increased doxorubicin fluorescence in PaLung and PaKiT03 cells (Fig. 3a, b and Supplementary Fig. 3). Likewise, pre-treatment with cyclosporin A (CSA), another ABC transporter inhibitor ${ }^{32}$, also led to an increase in doxorubicin 
a
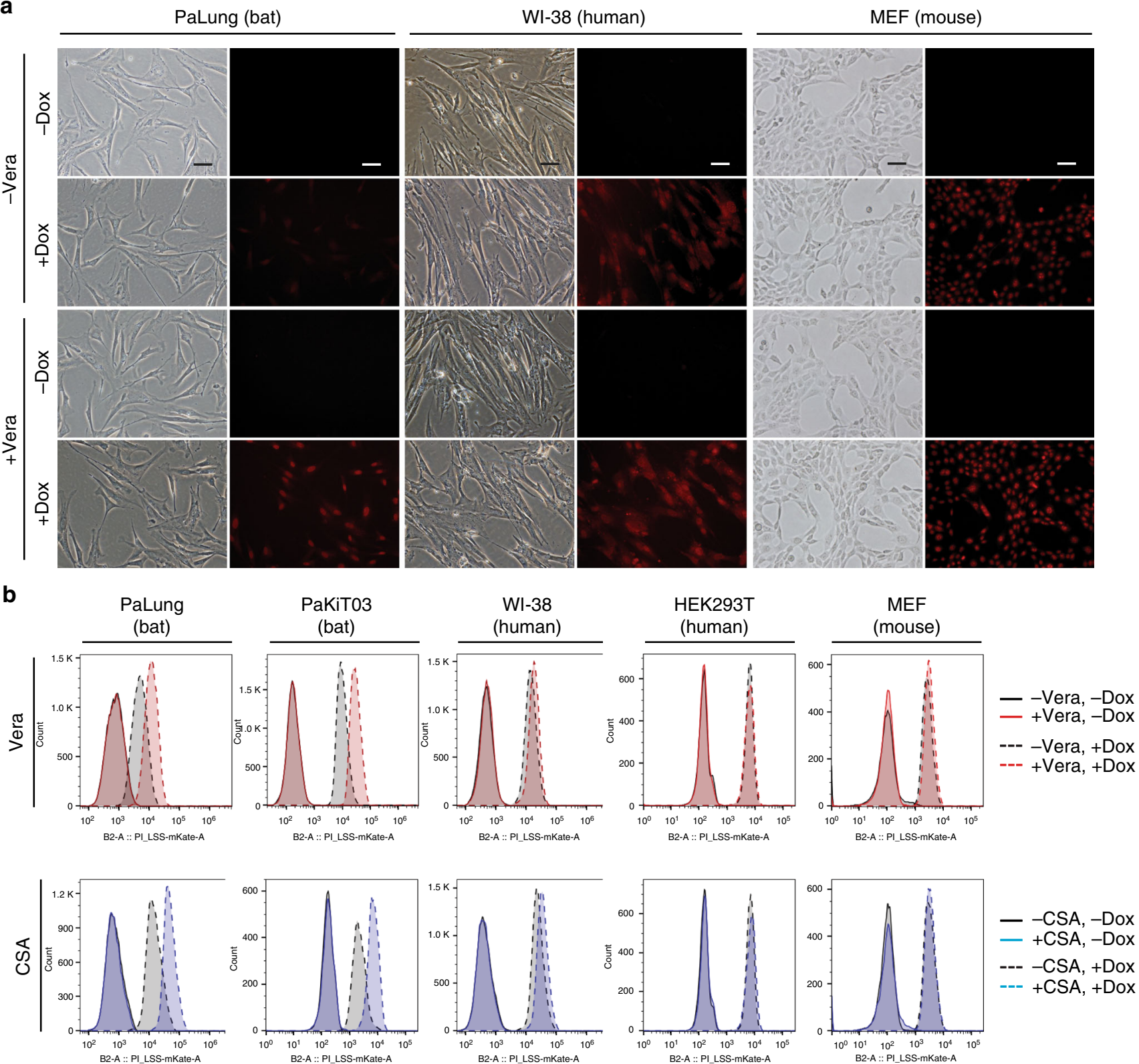

C
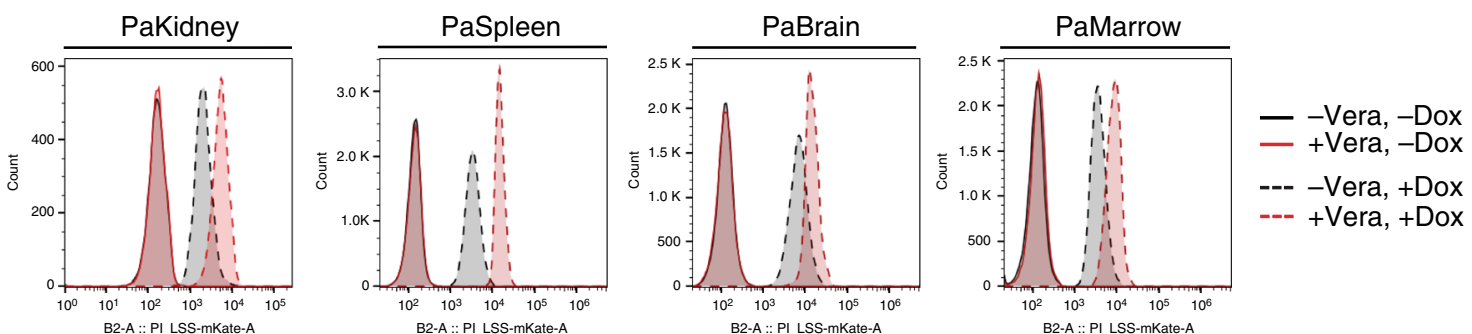

Fig. 3 Analysis of drug efflux capability via ABC transporters. a Doxorubicin (Dox) fluorescence or phase contrast images of PaLung, WI-38, and MEF cells. Cells were pre-treated without or with $5 \mu \mathrm{M}$ verapamil (Vera) for 30 min before the treatment with $10 \mu \mathrm{M}$ doxorubicin alone or together with Vera for an additional $3 \mathrm{~h}$. Images were acquired with $20 \times$ objective lens, and scale bar represents $50 \mu \mathrm{m}$. b Flow cytometry analyses of Dox accumulation in PaLung, PaKiT03, WI-38, HEK293T, and MEF cells. Cells were pre-treated without or with $5 \mu \mathrm{M}$ verapamil (Vera, top row) or $5 \mu \mathrm{M}$ cyclosporin A (CSA, bottom row) for 30 min before the treatment with $10 \mu \mathrm{M}$ doxorubicin alone or together with Vera or CSA for an additional $3 \mathrm{~h}$. c Flow cytometry analyses of Dox accumulation in cells derived from different tissues of $P$. alecto in the presence or absence of verapamil. Cells were treated and analysed as in (b). All results shown are representative of at least three experimental repeats 
fluorescence in PaLung and PaKiT03 cells, but not in WI-38, HEK293T, and MEF cells (Fig. 3b). These results suggest that the drug efflux activity of $\mathrm{ABC}$ transporters is required to reduce doxorubicin accumulation in PaLung and PaKiT03 cells. Furthermore, primary $P$. alecto cell lines derived from kidney (PaKidney), spleen (PaSpleen), brain (PaBrain), and bone marrow (PaMarrow) also displayed a similar increase in doxorubicin accumulation with verapamil pre-treatment (Fig. 3c). Together, these results suggest that cells derived from multiple tissues of $P$. alecto possess drug efflux activity via $\mathrm{ABC}$ transporters.

Drug efflux in bat cells reduces the exposure to doxorubicin genotoxicity. We hypothesised that $\mathrm{ABC}$ transporter activity underlies the increased resistance of bat cells to chemotherapeutic drugs. This hypothesis predicts that pre-treatment with inhibitors of $\mathrm{ABC}$ transporters will increase the genotoxic effects of doxorubicin in P. alecto cells but not in WI-38, HEK293T and MEF cells, which lack substantial ABC transporter activity. Indeed, verapamil pre-treatment enhanced the induction of $\gamma \mathrm{H} 2 \mathrm{AX}$ levels by low dose of doxorubicin $(1 \mu \mathrm{M})$ in PaLung and PaKiT03 cells, but not in WI-38, HEK293T and MEF cells (Fig. 4a, lanes 1, 2, 5 and 6), confirming that the $\mathrm{ABC}$ transporter activity helps to prevent DNA damage in PaLung and PaKiT03 cells. Treating PaLung and PaKiT03 cells with higher doses (5 and $10 \mu \mathrm{M})$ of doxorubicin in the presence of verapamil led to a doxorubicin dose-dependent decrease in $\gamma \mathrm{H} 2 \mathrm{AX}$ levels (Fig. 4a, lanes 6-8), as observed in WI-38, HEK293T and MEF cells with or without

a

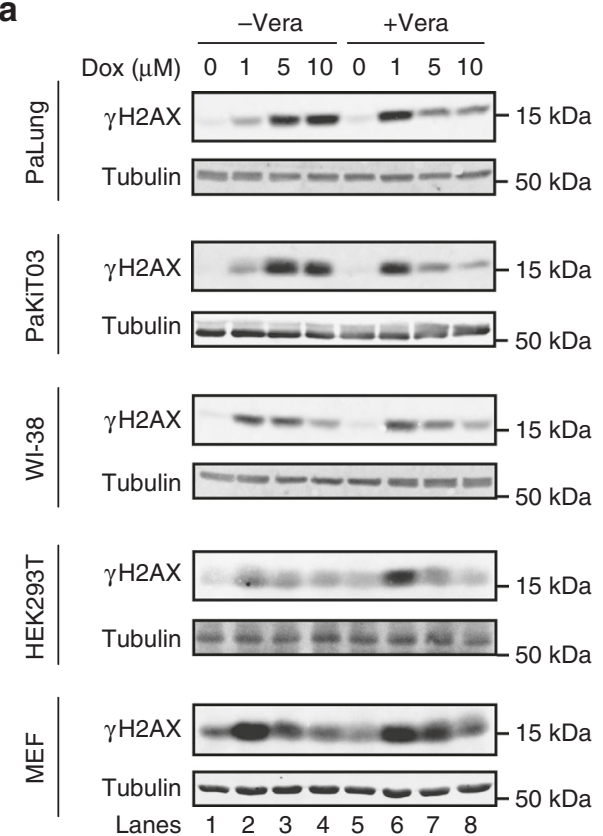

C

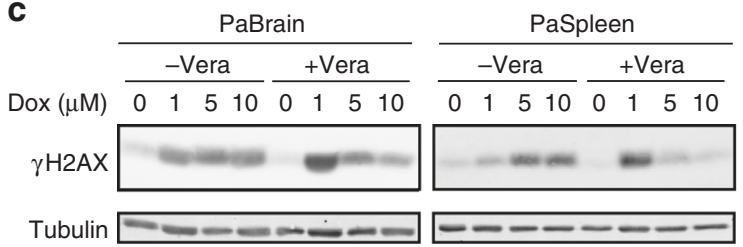

verapamil pre-treatment, and consistent with histone eviction ${ }^{30}$ (Fig. 4a, lanes 2-4 and 6-8). Similar results were obtained with the ABC transporter inhibitor CSA (Fig. 4b) and with cells derived from different tissues of $P$. alecto (Fig. 4c). Together, these results demonstrate that broad and efficient drug efflux in $P$. alecto cells contributes to reducing doxorubicin-mediated genotoxicity.

$\mathrm{ABCB} 1$ is responsible for increased drug efflux in bat cells. To determine the transporter(s) responsible for the efflux of doxorubicin in bat cells, we re-analysed our previously published genome-wide transcriptome data set of $P$. alecto kidney cell line PaKiT03 ${ }^{33}$. We identified three highly expressed ABC transporters (ABCB1, ABCC1 and ABCG2) that are well-characterised for doxorubicin efflux (Fig. 5a) ${ }^{32,34}$. To determine if one of these transporters is required for doxorubicin efflux in $P$. alecto cells, we reduced the expression of each gene in PaKiT03 cells via small-interfering RNA (siRNA)-mediated knockdown (Fig. 5b) and examined the effects on intracellular doxorubicin accumulation by flow cytometry (Fig. 5c). Verapamil was used as a positive control for efflux inhibition. We found that only $A B C B 1$ knockdown increased doxorubicin accumulation to a similar extent as verapamil pre-treatment, suggesting that ABCB1 is the transporter responsible for doxorubicin efflux in $P$. alecto-derived cells.

To further verify our observation, we treated WI-38 and PaLung cells with cisplatin, which is not an efflux substrate of

b
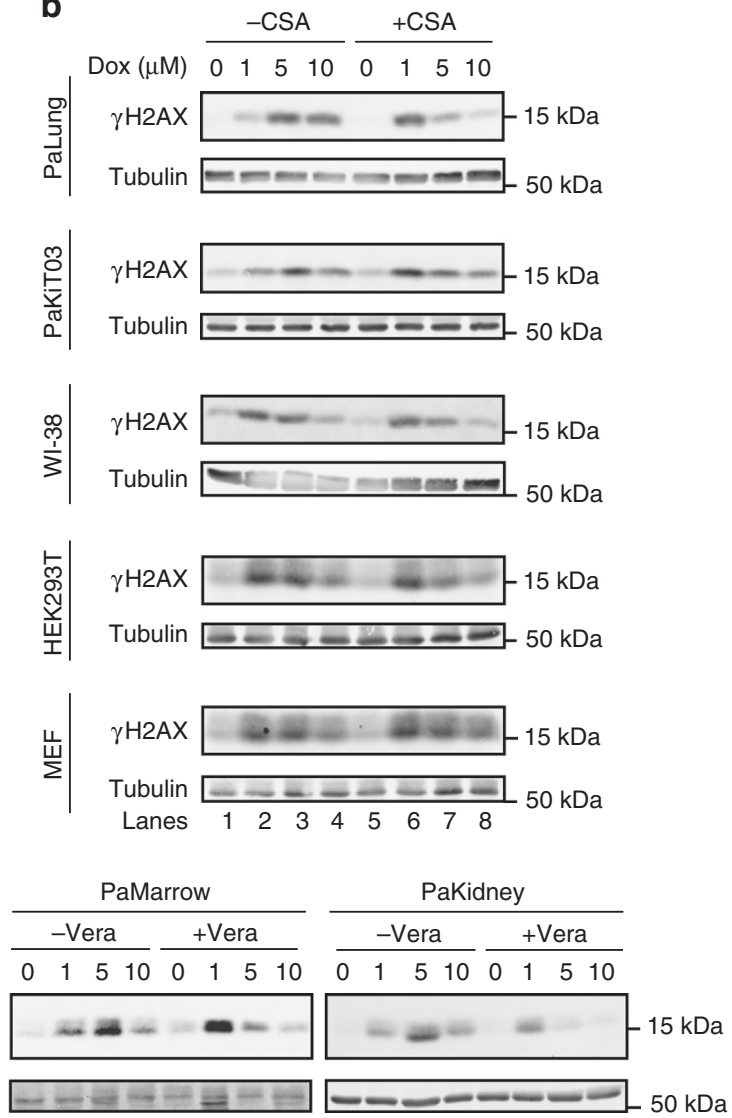

Fig. 4 Effect of $A B C$ transporter inhibition on $\gamma \mathrm{H} 2 \mathrm{AX}$ induced by doxorubicin. $\mathbf{a}$, $\mathbf{b}$ Western blot analyses of $\gamma \mathrm{H} 2 \mathrm{AX}$ induced by increasing doses of doxorubicin (Dox). PaLung, PaKiT03, WI-38, HEK293T and MEF cells were pre-treated without or with $5 \mu$ M verapamil (Vera) (a) or $5 \mu$ M cyclosporin A (CSA) (b) for 30 min before the treatment with the increasing amount of DOX alone or together with Vera or CSA for additional $3 \mathrm{~h}$. c Cells derived from different tissues of $P$. alecto were treated as in (a) and analysed by Western blotting. All results are representative of at least three experimental repeats. Tubulin was used as a loading control 


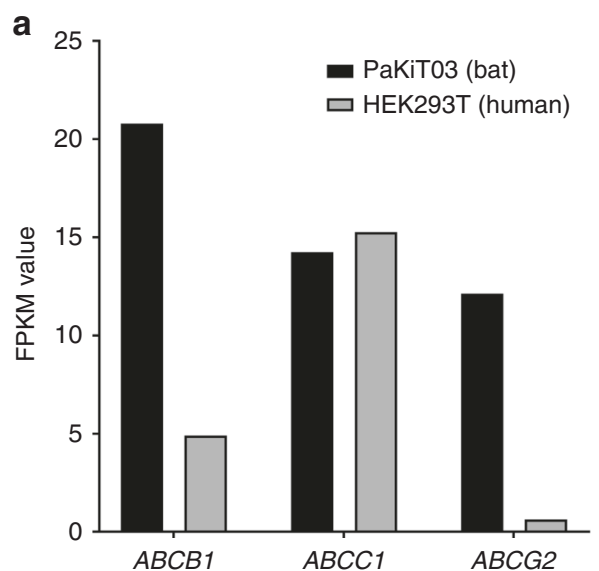

C

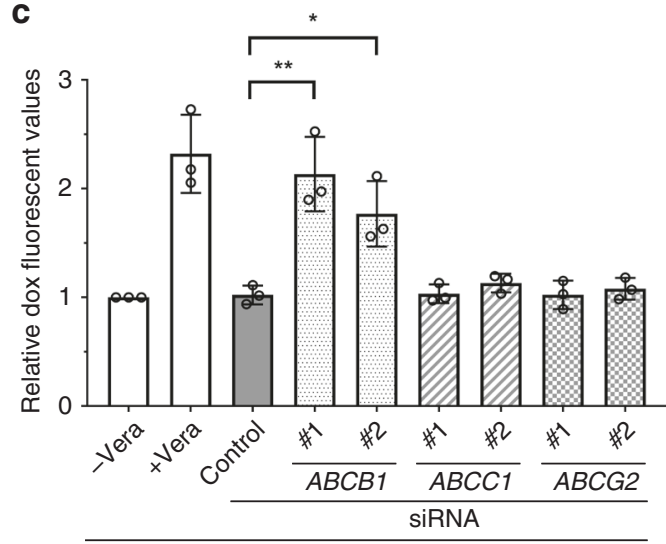

b

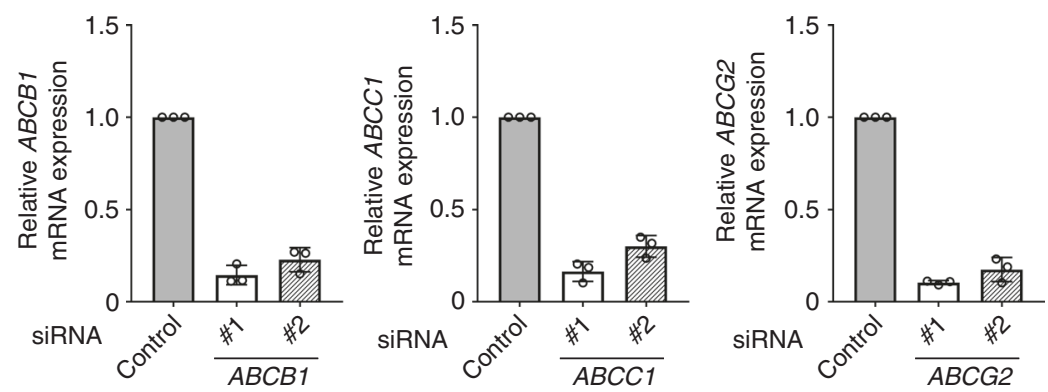

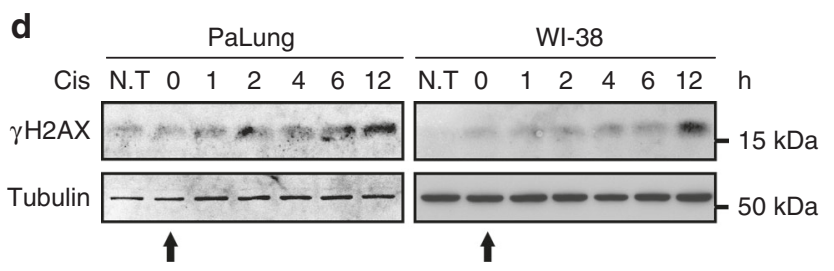

$10 \mu \mathrm{M}$ Dox

Fig. 5 Identification of $A B C B 1$ for the intrinsic doxorubicin efflux capability in bat cells. a Relative mRNA expression levels of $A B C B 1, A B C C 1$ and $A B C G 2$ in human HEK293T and P. alecto kidney immortalised (PaKiT03) cell lines are shown by FPKM values. $\mathbf{b}$ qRT-PCR to determine the knockdown efficiency of each ABC transporter. PaKiT03 cells were transfected with control or two independent siRNAs of the indicated genes. mRNA expression of each gene is normalised to GAPDH mRNA expression, and presented relative to the control siRNA sample (mean \pm SD from three experiments). c Doxorubicin (Dox) accumulation in PaKiTO3 cells after siRNA knockdown of $A B C B 1, A B C C 1$ and $A B C G 2$. Cells were treated with $10 \mu \mathrm{M}$ doxorubicin for $3 \mathrm{~h}$ and analysed by flow cytometry. Verapamil (Vera) was used as a positive control for $A B C$ transporter inhibition. Control represents the cells transfected with control siRNA. "Relative values" represents fluorescence intensities of the indicated condition relative to the fluorescent intensity of cells treated Dox alone ("-Vera", the first bar) (mean \pm SD from three experiments). Statistical significances were calculated using unpaired student's two-sided $t$ test. $p<0.05$ is represented with ${ }^{\star}$ and $p<0.01$ with ${ }^{*}$. d Western blot analysis of $\gamma \mathrm{H} 2 \mathrm{AX}$ in PaLung and WI-38 cells at different time points after a 3-h treatment with $10 \mu \mathrm{g} / \mathrm{ml}$ cisplatin (Cis). N.T stands for no treatment (starting at $t=0 \mathrm{~h}$, indicated by arrow). The results are representative of three independent experiments.

Tubulin was used as a loading control

$\mathrm{ABCB} 1^{35,36}$, and assessed $\gamma \mathrm{H} 2 \mathrm{AX}$ levels over time after drug removal. We found that WI-38 and PaLung cells treated with cisplatin displayed similar $\gamma \mathrm{H} 2 \mathrm{AX}$ induction (Fig. 5d), suggesting that both WI-38 and PaLung cells were equally exposed to the genotoxicity of cisplatin. In contrast, $\gamma \mathrm{H} 2 \mathrm{AX}$ induction by etoposide, which is an efflux substrate of $\mathrm{ABCB} 1^{34}$, resolved earlier in PaLung cells than in WI-38 and MEF cells (Fig. 1c), suggesting that ABCB1-mediated efflux of etoposide reduces its genotoxicity in PaLung cells.

ABCB1 protects bat cells from DNA damage and cell death. To assess whether $\mathrm{ABCB} 1$ protects $P$. alecto cells from DNA damage, we used comet assay to directly measure doxorubicin-induced DNA breaks in PaKiT03 cells, with or without siRNA-mediated depletion of $A B C B 1$ (Fig. 6a). Knockdown of $A B C B 1$ significantly increased the DNA damage caused by doxorubicin, as shown by the length of comet tails and the quantified olive tail moments (Fig. 6b, c). Consistent with these observations, verapamil pretreatment of PaKiT03 cells also increased the DNA damage caused by doxorubicin (Fig. 6d, e), supporting the conclusion that
ABCB1 helps to prevent the DNA damage induced by doxorubicin in $P$. alecto cells.

In addition, we evaluated the effect of $\mathrm{ABCB} 1$ inhibition on the viability of $P$. alecto cells treated with doxorubicin. We cultured PaKiT03 cells in media ( + or - verapamil) with doxorubicin for $3 \mathrm{~h}$, followed by doxorubicin-free media for an additional $72 \mathrm{~h}$. We then assessed cell viability by measuring ATP levels. We found that ABCB1 inhibition significantly reduced the viability of PaKiT03 cells exposed to doxorubicin, further suggesting that ABCB1 transport activity protects cells from genotoxic stress (Fig. 6f).

ABCB1 is highly and broadly expressed in $P$. alecto. To determine if $A B C B 1$ is differentially expressed in P. alecto compared to human cells, we first performed RT-qPCR on a panel of cell lines. Consistent with our RNA-seq results from PaKiT03 and HEK293T cells (Fig. 5a), ABCB1 transcript levels were significantly higher in various $P$. alecto cell lines than in various human cell lines (Fig. 7a). Further, ABCB1 protein levels were higher in PaLung and PaKiT03 cells compared to human WI-38 
a

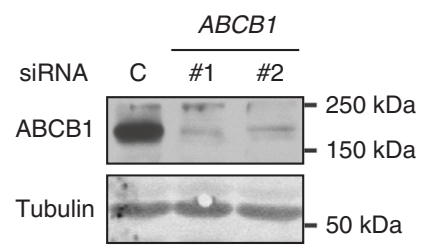

b

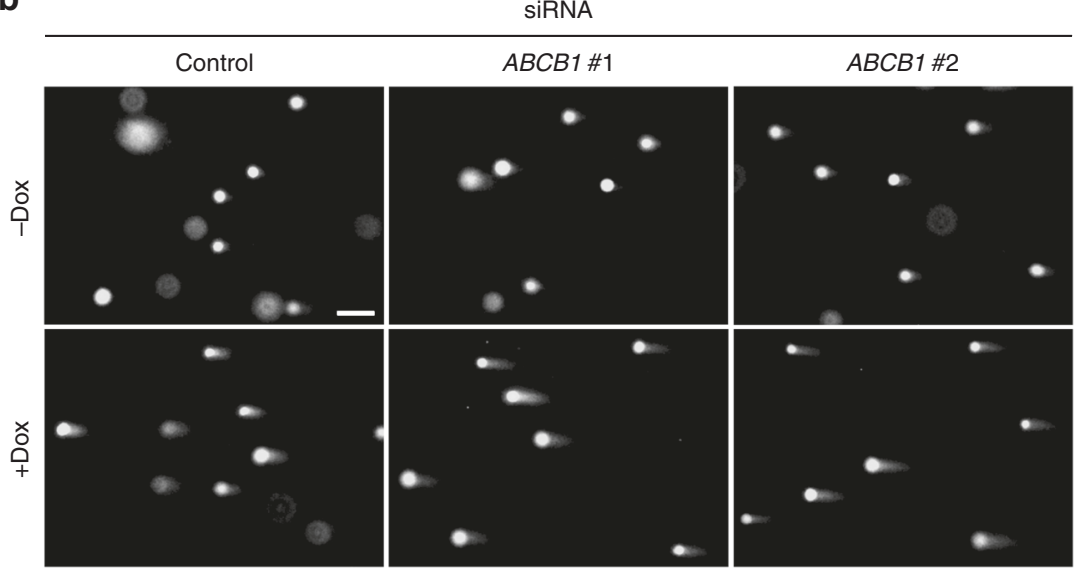

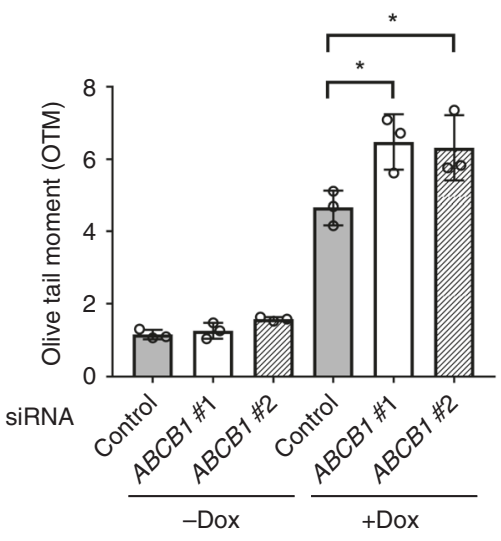

e

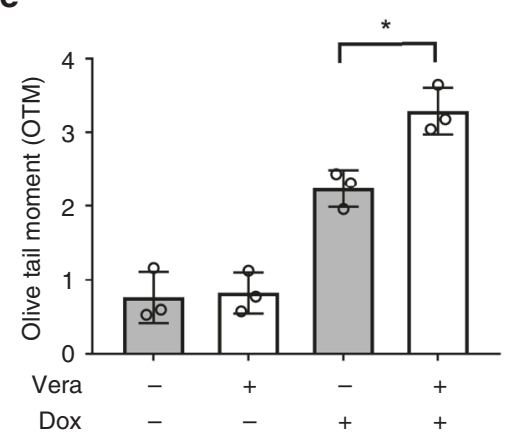

d
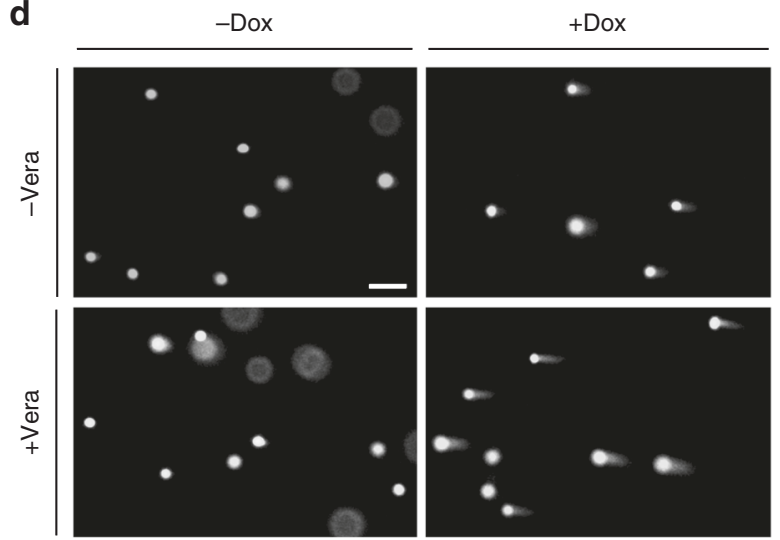

f

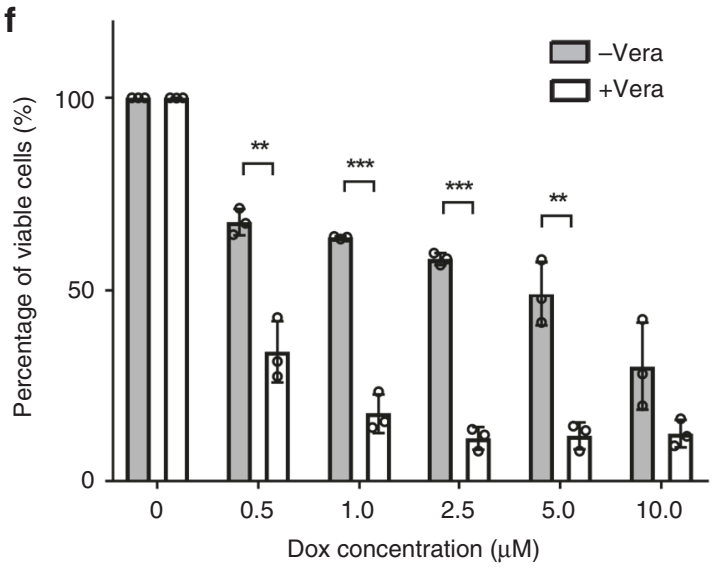

Fig. 6 Effect of $A B C B 1$ knockdown on doxorubicin-induced DNA damage in bat cells. a The efficiency of siRNA knockdown. PaKiT03 cells were transfected with the indicated siRNA, and the expression of $A B C B 1$ was analysed by Western blotting. C; control siRNA. Tubulin was used as a loading control. b Comet assay of PaKiT03 cells. siRNA knockdown cells were treated without or with $10 \mu \mathrm{M}$ doxorubicin (Dox) for $3 \mathrm{~h}$, and DNA damage was visualised by comet assay. c Quantification of the amount of DNA damage calculated by olive tail moments (OTM) for the cells in (b). d Comet assay of PaKiTO3 cells. Cells were pre-treated without or with $5 \mu \mathrm{M}$ verapamil (Vera) for 30 min before the treatment with $10 \mu \mathrm{M}$ doxorubicin alone or together with Vera for an additional $3 \mathrm{~h}$. DNA damage was visualised by comet assay. e Quantification of the amount of DNA damage calculated by OTM for the cells in (d). f Quantification of cell viability. Cells were pre-treated without or with $5 \mu \mathrm{M}$ verapamil for 30 min before the treatment with the indicated amount of doxorubicin alone or together with verapamil for an additional $3 \mathrm{~h}$. Doxorubicin was then removed and replaced with the doxorubicin-free medium in the absence or presence of verapamil for $72 \mathrm{~h}$ before measuring cell viability. Cell viability (\%) is presented relative to the control cells (the first grey bar). All data shown have at least three experimental repeats and the images are representative. Scale bar represents $50 \mu \mathrm{m}$. Bars represent the means \pm SDs of three independent experiments. Statistical significances were calculated using unpaired student's two-sided $t$ test. $p<0.05$ is represented with *, $p<0.01$ with ${ }^{\star *}$ and $p<0.001$ with $* \star \star$ 
a

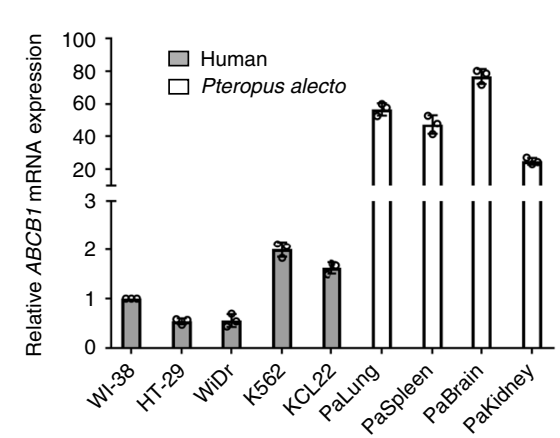

b

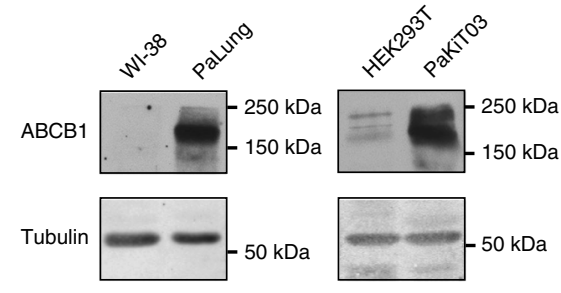

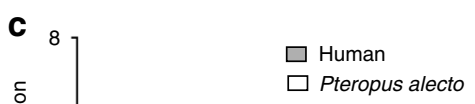

d
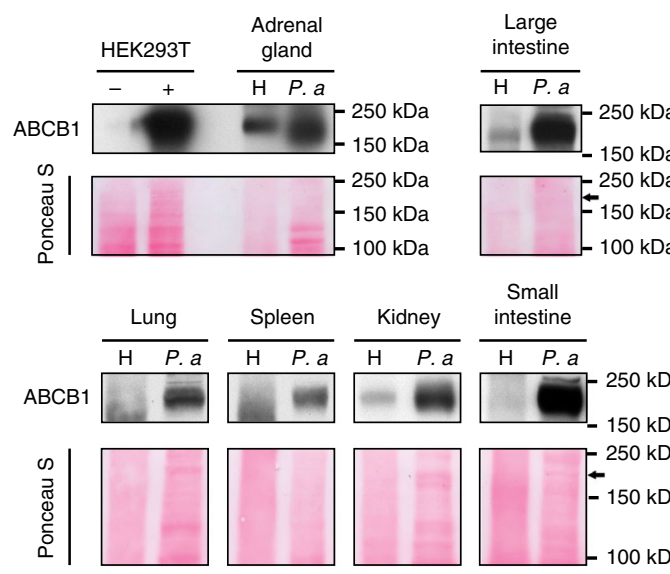

intestine

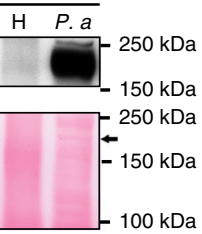

f
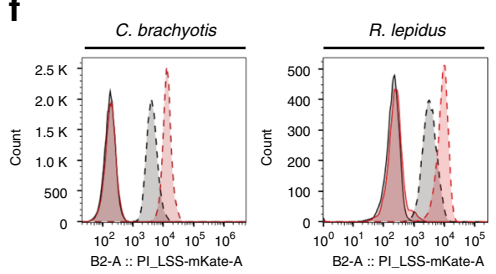

g
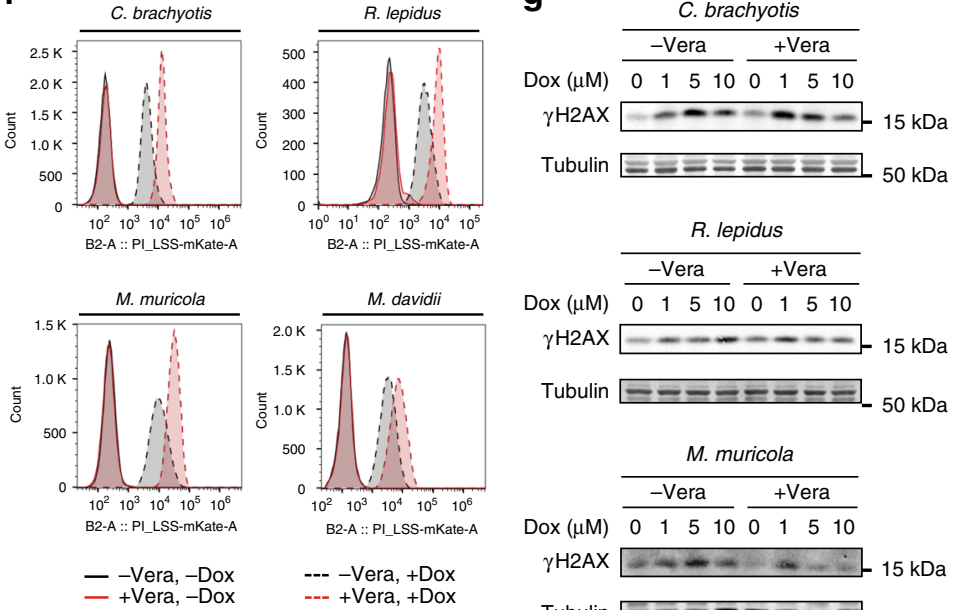

$\operatorname{Dox}(\mu \mathrm{M}) \quad \begin{array}{lllllllll}0 & 1 & 5 & 10 & 0 & 1 & 5 & 10\end{array}$

$\gamma \mathrm{H} 2 \mathrm{AX}$

Tubulin $=-=-=-50 \mathrm{kDa}$

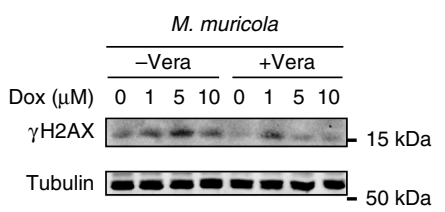

h

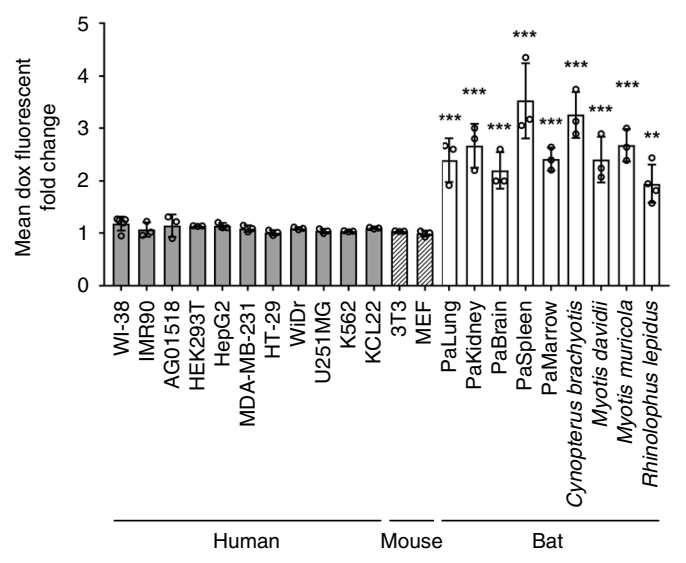

Fig. 7 ABCB1 expression and efflux capability in human and multiple bat species. a qRT-PCR for $A B C B 1$ mRNA expression in cell lines derived from human and $P$. alecto. $A B C B 1$ mRNA expression is normalised to GAPDH and presented relative to the expression in WI-38 cells (mean \pm SDs of three independent experiments). $\mathbf{b}$ Western blot analysis of $A B C B 1$ in the indicated cell lines. Tubulin was used as a loading control. c $q R T-P C R$ for $A B C B 1$ mRNA expression in tissues derived from human $(N=1)$ and $P$. alecto $(N=3)$. $A B C B 1$ mRNA expression is normalised to $G A P D H$ and presented relative to the respective human tissues (mean \pm SDs). $\mathbf{d}$ Western blot analysis of $A B C B 1$ protein in the indicated tissues. Ponceau $S$ staining of the membrane is shown as a loading control. Arrow indicates the size of $A B C B 1$ protein. $\mathrm{H}$ and $\mathrm{P}$. a indicate human and P. alecto respectively. Lysates from HEK293T transfected with control ( - ) or $A B C B 1$ expressing plasmid $(+)$ serve as negative and positive control, respectively. e Western blot analysis of ABCB1 in cells derived from the different bat species. Actin was used as a loading control. $\mathbf{f}$ Flow cytometry analysis of doxorubicin accumulation in cells derived from different bat species. Cells were treated and analysed as in Fig. 3b. $\mathbf{g}$ Western blot analyses of $\gamma \mathrm{H} 2 \mathrm{AX}$ in cells derived from different bat species. Cells were treated as in Fig. 4. Blots are representative of three independent experiments. $\mathbf{h}$ Flow cytometry analysis of doxorubicin accumulation. Cells were pre-treated without or with $5 \mu \mathrm{M}$ verapamil (Vera) for 30 min before the treatment with $10 \mu \mathrm{M}$ doxorubicin alone or together with Vera for an additional $3 \mathrm{~h}$. The fold change by Vera treatment is presented as the mean fluorescence intensity of cells treated with both verapamil and doxorubicin relative to the mean fluorescence intensity of cells treated with doxorubicin alone. Bars represent mean fold change \pm SD of at least three experiments. Data shown in Figs. $3 \mathrm{~b}, \mathrm{c}$ and $7 \mathrm{f}$ are also included in this histogram. Statistical significances were calculated using unpaired student's two-sided $t$ test. ${ }^{\star \star} p<0.01,{ }^{\star \star \star} p<0.001$ 
and HEK293T cells (Fig. 7b). Next, we examined ABCB1 transcript and protein levels in various normal tissues from $P$. alecto and human. We found that ABCB1 levels are higher in $P$. alecto than in human across multiple tissues (Fig. 7c, d), including tissues known to express ABCB1 in human (adrenal gland, kidney, small intestine and large intestine) as well as those that express no or very little $\mathrm{ABCB} 1$ in human (lung and spleen). These data suggest that $\mathrm{ABCB} 1$ is expressed at higher levels and more broadly in $P$. alecto compared to human.

A comparison of $P$. alecto ABCB1 amino acid sequence with the human sequence demonstrated similarity of $89.5 \%$ (Supplementary Table 1). In addition, Walker A-, Walker B-, and active transport signature-motifs, which are characteristic of $\mathrm{ABC}$ transporters, were $100 \%$ conserved across multiple phylogenetically distant mammals (Supplementary Fig. 4), indicating that the ABCB1 amino acid sequence is well-conserved among mammals. Therefore, the increased drug efflux via ABCB1 in P. alecto cells compared to human cells is likely due to the higher expression of $\mathrm{ABCB} 1$ rather than the amino acid sequence differences.

ABCB1-mediated efflux capability is conserved in multiple bat species. To determine if other families of bats also possess ABCB1-dependent drug efflux, we investigated cells derived from Cynopterus brachyotis (Pteropodidae family), Rhinolophus lepidus (Rhinolophidae family) and Myotis muricola and Myotis davidii (Vespertilionidae family), which have distinct maximum lifespans and body masses (Supplementary Table 2). Cells from these bat species showed high expression of ABCB1 protein, similar to $P$. alecto (Pteropodidae family) (Fig. 7e), as well as efficient doxorubicin efflux that depended on ABC transporter activity (Fig. 7f). Further, these cells displayed the induction of $\gamma \mathrm{H} 2 \mathrm{AX}$ by doxorubicin, but pre-treatment with verapamil led to a doxorubicin dose-dependent decrease in $\gamma \mathrm{H} 2 \mathrm{AX}$ levels (Fig. $7 \mathrm{~g}$ ), consistent with elevated levels of intracellular doxorubicin and histone eviction upon inhibition of ABCB1.

To further confirm that $\mathrm{ABCB} 1$-mediated drug efflux is unique to bats, we extended our analysis to a diverse range of cell lines from bats, mice, and humans. We examined doxorubicin accumulation in the presence or absence of verapamil by flow cytometry in 11 human cell lines, 2 mouse cell lines, 5 P. alecto cell lines, and 4 cell lines from other bat species (Supplementary Table 3). Verapamil pre-treatment increased doxorubicin accumulation in all of the bat cell lines, but not in any of the human and mouse cell lines (Fig. 7h). Together, these results suggest that high $\mathrm{ABCB} 1$ expression and enhanced drug efflux capacity are uniquely conserved in multiple bat species and may contribute to reducing DNA damage and perhaps, in turn, cancer incidence in bats.

\section{Discussion}

Emerging evidence suggests that several long-lived mammals possess unusually low rates of cancer occurrence ${ }^{2}$. Technological advances in genomic sequencing have facilitated analysis of these organisms and revealed unique mechanisms of cancer resistance that are not observed in human ${ }^{2}$. Bats are long-lived mammals with an extremely low incidence of cancer $^{2,10}$. However, the mechanisms underlying their cancer resistance are not fully understood. We have access to the bat species P. alecto, a large, non-hibernating fruit bat of the Pteropodidae family. Using cell lines established from $P$. alecto, we discovered that $P$. alecto cells uniquely displayed increased resistance to DNA damage induced by specific chemicals. This resistance is due at least in part to increased efflux of the chemicals mediated by high and broad expression of the ABCB1 transporter.
ABC transporter family proteins regulate cellular drug efflux ${ }^{32}$. $\mathrm{ABCB} 1$, also known as the transmembrane protein $\mathrm{P}$ glycoprotein (P-gp) and multidrug resistance protein 1 (MDR1), is one of the best-characterised efflux transporters and is highly conserved across mammals ${ }^{37}$. In mammals, ABCB1 is specifically expressed in regions of detoxification, excretion, and protective barriers such as the intestinal epithelium, lumens of the liver, proximal tubule of the kidney as well as blood-tissue barriers of the brain. In rodents, the $A B C B 1$ gene encodes two isoforms, $A b c b 1 a$ (Mdr1a) and $A b c b 1 b(M d r 1 b)$. Mice deleted for $A b c b 1 a$ alone or both $A b c b 1 a$ and $A b c b 1 b$ are viable and not cancer-prone in laboratory conditions, which are free from genotoxic substances. However, they are more sensitive to toxins than wild-type mice ${ }^{38,39}$. We found that $P$. alecto expresses ABCB1 relatively highly and in additional tissues compared to human, such as lung and spleen. The broad expression of ABCB1 may contribute to more efficient protection from genotoxic substances in their environment.

$\mathrm{ABCB} 1$ was originally discovered in cancer cells where its high and functional expression promotes resistance to a variety of chemotherapeutic drugs, such as paclitaxel, vinblastine, etoposide and doxorubicin ${ }^{32,34,40}$. This broad chemoresistance is achieved by the promiscuous substrate specificity of ABCB1. Cancer cells acquire $\mathrm{ABCB} 1$ overexpression after prolonged chemotherapy, which is one of the major causes of cancer relapse since ABCB1mediated drug efflux can reduce drug intake ${ }^{32,34}$. Likewise, generating cancer cell lines with high ABCB1 expression to study the biological implications of $\mathrm{ABCB} 1$ often requires prolonged exposure of cells to drugs for months ${ }^{41,42}$. It is, therefore, rather intriguing that various bat cell lines from many different tissues intrinsically possess and maintain high-ABCB1 expression and drug efflux in the absence of known selective pressure. Notably, in addition to xenobiotics, ABCB1 is reported to transport endogenous compounds such as cytokines, short peptide and lipids ${ }^{43,44}$. It is possible that bat cells may possess endogenous substances that are ABCB1 efflux substrates, and thus express high levels of ABCB1 to maintain homoeostasis.

$\mathrm{ABCB} 1$ expression is regulated by multiple signalling pathways and physiological factors. Previous studies have reported that cMyc, c-Jun, HIF-1 and CtBP1 can positively modulate ABCB1 expression in cancers, leading to the development of drug resistance ${ }^{45}$. Recent work on microRNA biology (miRNA) such as miR-27a ${ }^{46,47}$, miR-223 $3^{48}$ and miR-451 ${ }^{46}$, have also illustrated their potential roles in regulating $\mathrm{ABCB} 1$ expression. Furthermore, physiological substances including steroid hormones, environmental stresses such as temperature, osmotic pressure and $\mathrm{pH}$, and xenobiotics such as insecticides, heavy metal and DNA damage reagents have been shown to significantly modulate $\mathrm{ABCB} 1$ expression in vitro ${ }^{45}$. Interestingly, human pathogenic virus and virus proteins are also reported to induce $A B C B 1$ expression ${ }^{49,50}$. Since bats are renowned for being a reservoir of high mortality viruses like Ebola ${ }^{51}$ and SARS $^{52}$, some viral proteins may induce $\mathrm{ABCB} 1$ expression. It remains to be elucidated how bat cells induce and retain the high expression of ABCB1.

High doses of $\gamma$-irradiation, such as $10 \mathrm{~Gy}$ used in this study, mainly cause DNA DSB ${ }^{53}$. DSBs are the most detrimental form of DNA breaks for cells, and most DSBs are repaired via nonhomologous end-joining (NHEJ) ${ }^{26,54} \cdot \gamma \mathrm{H} 2 \mathrm{AX}$ and 53BP1 protein are core components of the NHEJ pathway. Once phosphorylation of $\mathrm{H} 2 \mathrm{AX}$ occurs in response to DSBs, 53BP1 rapidly forms foci and disappears upon repair. We observed that 53BP1 foci formation and clearance after $10 \mathrm{~Gy}$ of $\gamma$-irradiation were fairly comparable between the cells from $P$. alecto and human, whereas mouse cell displayed a slower 53BP1 response. On the other hand, $\gamma \mathrm{H} 2 \mathrm{AX}$ signals induced by the same treatment were similar between bat, human and mouse cells. Why there is a delay 
between $\gamma \mathrm{H} 2 \mathrm{AX}$ phosphorylation and 53BP1 foci formation in mouse cells compared to human and bat cells remains to be deciphered.

Nucleotide excision repair (NER) and base excision repair (BER) are utilised to repair single-strand breaks such as removing bulky DNA adducts caused by UV irradiation ${ }^{55}$. Podlutsky et al. ${ }^{56}$ reported that human and bat cells have better BER than mouse cells, whereas human cells have better NER than bat and mouse cells. Our data suggest that human and bat cells have a better response to DSBs compared to mouse cells. It is likely that each species has evolved different strategies to cope with the different types of DNA damage.

Doxorubicin was originally isolated from a soil bacterium and can induce DSBs and single-stranded breaks via multiple mechanisms, including DNA intercalating to inhibit topoisomerase II activity, histone eviction, and induction of oxidative stress ${ }^{30,57}$. The mechanisms of cell death induced by doxorubicin are cell context-dependent and could be mediated by DNA damage or be independent of DNA damage such as replication stress or oxidative stress to proteins and other organelles ${ }^{57}$. Importantly, our data show that bat cells accumulate less doxorubicin compared to human and mouse cells, leading to reduced $\mathrm{DSBs}$, and that $\mathrm{ABCB} 1$ is responsible for the reduced doxorubicin accumulation in bat cells.

Etoposide was originally isolated from plants and forms a ternary complex with DNA and topoisomerase II, thereby causing DNA DSBs ${ }^{29}$. In our experiments, we found that $\gamma \mathrm{H} 2 \mathrm{AX}$ resolved faster in $P$. alecto cells than in human and mouse cells when transiently treated with etoposide. Etoposide is a well-known ABCB1 substrate, therefore human and mouse cells would be expected to have higher residual intracellular etoposide than $P$. alecto cells even after drug removal from the media, leading to more DNA damage and $\gamma \mathrm{H} 2 \mathrm{AX}$ accumulation than $P$. alecto cells. Thus, the prolonged $\gamma \mathrm{H} 2 \mathrm{AX}$ in human and mouse cells could reflect not only DNA repair but also DNA damage caused by residual etoposide. Similar to doxorubicin, the assessment of DNA repair caused by etoposide between the $P$. alecto, human and mouse cells is challenging since the amounts of intracellular etoposide are not equal among these animals.

Other mechanisms of cancer resistance in bats have been proposed previously. For example, genome studies of the longestlived bat Myotis brandtii showed that cancer resistance might be attributed to growth suppression by specific mutations in the growth hormone receptor that may reduce the GH-insulin-like growth factor 1 signalling pathway ${ }^{58}$. In addition, miRNA analysis revealed that three tumour suppressive miRNAs (miR-101$3 p$, miR-16-5p and miR-143-3p) were upregulated and a single tumour promoting miRNA (miR-221-5p) was downregulated in Myotis myoti ${ }^{59}$. These data suggest that bats may have multiple strategies to prevent tumour formation.

Unlike conventional mouse models, in vivo validation of our proposed mechanism in bats is hindered by their low reproductive capacity and technological limitations for genetic manipulation ${ }^{10,60}$. Since the function of $A B C B 1$ in mice was reported to be limited to specific tissues ${ }^{38,39}$, it would be of interest to determine whether transgenic mice with high and broad expression of $A B C B 1$ are less tumour prone, particularly in response to $A B C B 1$ substrates.

Laboratory-bred animals and their wild-caught counterparts likely respond differently to xenobiotic and environmental challenges ${ }^{61-63} . A B C B 1$ knockout mice are not cancer-prone in the laboratory conditions where is free from genotoxic substances but are more sensitive to toxins than wild-type mice ${ }^{39}$. Therefore, whether $A B C B 1$ knockout mice are cancer-prone in the wild remains to be investigated. Harper et al. have shown that cells derived from wild-trapped mice exhibited different sensitivity to various genotoxic insults than cells derived from laboratory-bred mice ${ }^{62}$. Hence, we do not exclude the possibility that the environmental background could contribute to the differences we observed between bats and mice. It would be interesting to compare the ABCB1-mediated efflux capacity of bat cells to cells derived from wild mice.

In summary, using $P$. alecto as a model, we demonstrated that bats have a high and broad expression of $A B C B 1$, which uniquely promotes resistance to DNA damage induced by specific chemicals. This phenotype is conserved in multiple bat species, possibly as a pan-bat biological feature, and might partly explain their low incidence of cancer. Future research into this potential tumour suppressor mechanism in bats could inform innovative strategies to prevent or treat human cancer, as well as chemoresistance.

\section{Methods}

Cell culture. P. alecto and M. davidii-derived cell lines listed in Supplementary Table 3 and were established as previously described ${ }^{18,64}$. Briefly, the tissues were first cut finely using a scalpel, washed with cold media and further processed by enzymatic reactions for primary cell culture. C. brachyotis, M. muricola and $R$. lepidus were captured in Singapore under the National Parks Board permit NP/ RP11-011 and NP/RP14-109. Lung tissues were harvested to produce primary cell cultures. Lung tissues were sliced into $1 \mathrm{~mm}$ sized pieces and treated enzymatically (1X HBSS, $0.2 \%$ bovine serum albumin, $0.4 \mathrm{mg} / \mathrm{mL}$ collagenase-A, $0.22 \mu \mathrm{m}$ filtered) and incubated at $37^{\circ} \mathrm{C}$ with rotation $(60 \mathrm{rpm})$ for approximately $1 \mathrm{~h}$ to dissociate cells. Dissociated tissues were centrifuged at $430 \times g$ for $5 \mathrm{~min}$, resuspended with complete RPMI medium 1640 (10\% foetal bovine serum (Thermo Scientific), 2\% penicillin/streptomycin (Thermo Scientific), 2.5\% HEPES) and plated in T-25 tissue culture flasks. Primary lung cells were maintained at $37^{\circ} \mathrm{C}$ with $5 \% \mathrm{CO}_{2}$ until confluence. After the second passaging, all cell lines were grown in Dulbecco's modified Eagle's medium (DMEM) with $10 \%$ foetal bovine serum and penicillin/ streptomycin in a $37^{\circ} \mathrm{C}$ incubator with $5 \% \mathrm{CO}_{2}$, with exception of $\mathrm{K} 562$ and KCL22 cells that were maintained in RPMI medium 1640 with $10 \%$ foetal bovine serum and penicillin/streptomycin. Primary cell lines from the tissues of other bat species were established using identical method for lung tissues. Origin of cell lines and immortalisation type are listed in Supplementary Table 3. Tissue samples of the adrenal gland, lung, spleen, kidney, small and large intestines were obtained from $P$. alecto and were stored in Buffer RLT (Qiagen) at $-80^{\circ} \mathrm{C}$ for protein extraction. WI-38, IMR-90 and AG01518 cell lines were purchased from Coriell Institute. HEK293T, HepG2, MDA-MB-231, HT-29, WiDr, U251MG, K562 and NIH3T3 cell lines were purchased from American Type Culture Collection (ATCC). KCL-22 cell line was purchased from Leibniz Institute DSMZ-German. MEF cells were kindly provided by Dr Yanping Zhang (University of North Carolina at Chapel Hill).

Reagents. Doxorubicin (D4035), etoposide (E1383), cisplatin (P4394), verapamil hydrochloride (V4629) and cyclosporin A (C3662) were obtained from SigmaAldrich.

Western blotting and antibodies. Protein extraction from cultured cells was conducted in sodium dodecyl sulfate (SDS) lysis buffer $(50 \mathrm{mM}$ Tris- $\mathrm{HCl} \mathrm{pH} 6.8$, $2 \%$ SDS, $10 \%$ glycerol). Protein extraction from $P$. alecto tissues was performed in RIPA lysis buffer (10 mM Tris-HCl pH 7.6, $150 \mathrm{mM} \mathrm{NaCl}, 1 \%$ TritonX-100, $1 \mathrm{mM}$ EDTA) supplemented with EDTA-free complete protease inhibitor cocktail (04693132001, Roche), phosphatase inhibitor cocktail 2 (P5726, Sigma-Aldrich), and phosphatase inhibitor cocktail 3 (P0044, Sigma-Aldrich) using cell pellet homogeniser (VWR) on ice. Protein lysates extracted from the human adrenal gland (sc-363761), lung (sc-363767), spleen (sc-363779), kidney (sc-363764), small intestine (sc-364225) and large intestine (sc-363757) were purchased from Santa Cruz biotechnology, Inc. Lysates were then quantified, and equal amounts of protein were loaded for Western blotting analyses. Antibodies used are as follow: $\gamma \mathrm{H} 2 \mathrm{AX}$ (05-636, Millipore, 1:3000), tubulin (ab44928, Abcam, 1:5000), actin (MAB1501, Millipore, 1:10,000), ABCB1 (sc-8313 H241, Santa Cruz, 1:1000), horseradish peroxidase (HRP)-conjugated secondary antibody (Thermo Scientific, 1:10,000), and fluorescence labelled secondary antibodies (Thermo Scientific, $1: 10,000)$. Detection of $\gamma \mathrm{H} 2 \mathrm{AX}$, tubulin and actin were performed using LI-COR Odyssey (LI-COR Biosciences) or HRP substrate (Thermo Scientific). ABCB1 was detected using HRP substrate (Thermo Scientific). Uncropped western blotting images are provided in the Supplementary Information.

Measurement of 53BP1 foci formation. Detection and quantification of 53BP1 foci formation were conducted using immunofluorescence and fluorescence microscopy. For immunofluorescence, PaLung, WI-38 and MEF cells were treated with either $10 \mathrm{~Gy}$ of $\gamma$-irradiation or $5 \mu \mathrm{M}$ doxorubicin for $3 \mathrm{~h}$ before subjected for analysis at the respective time points. In brief, cells were fixed with $4 \%$ 
formaldehyde (Sigma-Aldrich) and permeablized with 0.2\% Triton X-100 (BioRad) followed by staining with anti-53BP1 antibody (sc-22760, Santa Cruz, 1:250), Alexa Fluor 488-conjugated anti-rabbit IgG (Jackson ImmunoResearch, 1:100), and DAPI (Sigma-Aldrich). Imaging of cells was performed using Olympus IX83 fluorescent microscope at $405 \mathrm{~nm}$ for DAPI and $488 \mathrm{~nm}$ for 53BP1. As for quantification of 53BP1 foci, analysis of cells was carried out using cell image analysis software CelProfiler ${ }^{\mathrm{TM}}$ with the speckle counting pipeline ${ }^{65}$. A minimum of 100 cells was quantified per time point for each treatment condition. Average number of 53BP1 foci per cell was calculated using the equation: average number of 53BP1 foci per cell $=$ (total number of foci)/(total number of cells quantified).

\section{Measurement of intracellular doxorubicin accumulation. Accumulation of} intracellular doxorubicin was monitored or measured by intrinsic fluorescence of doxorubicin using either fluorescence microscopy or flow cytometry. For fluorescence microscopy, PaLung, PaKiT03, WI-38, HEK293T and MEF cells were pre-treated with or without $5 \mu \mathrm{M}$ verapamil for $30 \mathrm{~min}$, followed by incubation with doxorubicin for $3 \mathrm{~h}$. Detection of doxorubicin in live cells was performed using Olympus IX71S1F3 fluorescent microscope. As for flow cytometry analysis, cells were pre-treated with or without $5 \mu \mathrm{M}$ verapamil or cyclosporin A for $30 \mathrm{~min}$, followed by incubation with doxorubicin for $3 \mathrm{~h}$. Cells were then trypsinized, washed in PBS once and resuspended in PBS for analysis by MACSQuant Analyser 10 (Miltenyi Biotec). Laser excitation was set at 488 $\mathrm{nm}$ and filter with wavelengths $614 / 50 \mathrm{~nm}$ was used for detection. A total number of 30,000 live cells were analysed per sample. For Fig. 2e, f, and Supplementary Fig. $2 \mathrm{D}$ and $\mathrm{E}$, the calculation was done using the equation: normalised doxorubicin fluorescence value $=($ mean fluorescence value $) /$ (average cell volume). For Fig. 7h, the calculation was done using the equation: mean doxorubicin fluorescence fold change $=$ (mean fluorescence with efflux inhibition) $/$ (mean fluorescence without efflux inhibition).

Cell volume determination. Mean volume $(\mu \mathrm{L})$ of WI-38, PaLung, MEF, HEK293T and PaKiT03 cells were determined by Millipore Sceptre cell counter. Trypsinized cells were washed with PBS, before being measured using $60 \mu \mathrm{m}$ sceptre tips (Millipore).

Comet assay (DNA damage measurements in cells). DNA damage in PaKiT03 cells was assessed by neutral comet assay as described ${ }^{66}$. Comets were stained with propidium iodide for $30 \mathrm{~min}$ before subjected for analysis. Images were acquired with a $20 \times$ objective lens. A minimum of 50 comets was assessed per treatment condition. Analysis of the comets and DNA damage calculated by olive tail moments were performed using CASPlab software ${ }^{67}$. Olive tail moments were calculated using the formula: olive tail moments $=$ (the percentage of DNA in comet tail $) \times($ distance between comet head and tail centres of gravity).

Cell viability assay. PaKiT03 cells were seeded at 10,000 cells per well in a 96-well plate and kept overnight to allow attachment. Cells were pre-treated with or without $5 \mu \mathrm{M}$ verapamil for $30 \mathrm{~min}$, followed by co-treatment of increasing dose of doxorubicin $(0.5,1,2.5,5$ and $10 \mu \mathrm{M}$ doxorubicin) and verapamil for $3 \mathrm{~h}$. Doxorubicin was then removed, and cells were replaced in doxorubicin-free medium with or without verapamil for $72 \mathrm{~h}$ before measuring cell viability using CellTiter$\mathrm{Glo}^{\circ}$ luminescent cell viability assay purchased from Promega.

siRNA knockdown. Transient transfection of siRNAs was performed using ScreenFect A (Wako) according to manufacturer's instructions. Concentrations of siRNA for control, $A B C B 1, A B C C 1$ and $A B C G 2$ were set at $20 \mathrm{nM}$. Control siRNA was purchased from Integrated DNA Technologies (NC1). Sense strand sequence of the siRNAs for $A B C B 1, A B C C 1$ and $A B C G 2$ are as follows:

ABCB1 \#1 (5'-GAGCUUGAAAGGUACAACAAAAAdTdT-3'),

\#2 (5'-GAUGAAGCUACAUCAGCUCUAGAdTdA-3');

ABCC1 \#1 (5'-CAAACAGCAUCACCGUGAAAAACdGdC- $3^{\prime}$ ),

\#2 (5'-CUGGAAGAAGGAAUGUGCCAAAUdCdC-3');

ABCG2 \#1 (5'-CAUGAAUAUAUCAGUGGAUACUAdCdA-3'),

\#2 (5'-GGAGAAGAAUUCUUGAUAAAGCAdGdG-3').

Quantitative RT-PCR analysis. Total RNA from cell lines and tissue samples was extracted using TRIzol (Life Technologies) and RNeasy Mini Kit (Qiagen). A single panel of human tissue total RNA was purchased from Clontech (human total RNA Master Panel II \#636643) and bat tissue total RNA was harvested from three individual $P$. alecto. All cDNA was synthesised using iScript cDNA synthesis kit (BioRad). Quantitative RT-PCR was performed with SYBR Green (KAPA Biosystems) using the CFX96 System (BioRad). Relative expression of each gene was calculated using GAPDH as an internal control by BioRad CFX manager software. The primers used are as follows:

GAPDH forward (5'-CGTATTGGGCGCCTGGTCAC-3') and

reverse (5'-CATGTAGTTGAGGTCAATGAAGGGGTC-3');

$A B C B 1$ forward (5'-TGGTGTGGTGAGTCAGGAACCTG-3') and

reverse (5'-ACAGCTTTCTCAATCTCATCCATGGTG-3');
ABCC1 forward (5'-CCCATGAGGAGCTGGATCCGG-3') and reverse (5'-CTTCCGGAATGGAGAAGGTGATGC- ${ }^{\prime}$ );

ABCG2 forward (5'-GTGTGTCTTCTGTGGAGCTCTTTGTG-3') and

reverse (5'-CCCACCTCTGCTTTCAATCCTACC-3'). QPCR products were verified by sequencing.

RNAseq data analysis. Whole transcriptome shotgun sequencing data of PaKiT03 and HEK293T cells from our previous study was obtained from the NCBI Sequence Read Archive under the accessions [SRX661189] and [SRX665117] ${ }^{33}$. RNA-Seq reads were mapped using Tophat (version 1.3.2) and Bowtie (version 0.12.7). Fragments per kilobase million (FPKM) values were calculated using Cufflinks (version 1.2.1). Differential expression was calculated using DESeq2. FPKM values were normalised per gene to obtain relative expression values.

Protein sequence alignment and conservation comparison. Alignment of different mammalian ABCB1 protein sequences was generated with Bio Edit programme. Percentages of identity with other mammalian ABCB1 were analysed with reference to human $A B C B 1$ protein sequence using Vector NTI Advance version 11.5. In this study, amino acid sequences for ABCB1 were obtained for Bos taurus -cow (XP_010802430.1), Canis lupus familiaris - dog (NP_001003215.2), Felis catus-cat (NP_001164535.1), Heterocephalus glaber-naked mole rat (XP_012934158.1), Homo sapiens-human (NP_000918.2), Macaca mulattaRhesus monkey (NP_001028059.1), Mus musculus-mouse ABCB1A (NP 035206.2) and mouse ABCB1B (NP_035205.1), M. davidii-David's myotis (XP 006779228.1), P. alecto-black flying fox bat (XP_006925783) and Sus scrofa -pig (NP_001295175.1).

Database for different species of bats. Maximum lifespan and body mass of $P$ alecto, C. brachyotis were acquired from AnAge database of Animal Ageing and Longevity [http://genomics.senescence.info/species/]. For M. davidii, M. muricola and $R$. lepidus, maximum lifespan and body mass were determined by averaging Myotis genus and Rhinolophus genus, respectively using available information derived from the same database.

Statistical analysis. All statistical analyses in this study were conducted using the unpaired student's two-sided $t$ test.

Reporting summary. Further information on research design is available in the Nature Research Reporting Summary linked to this article.

\section{Data availability}

The RNA-seq of PaKiT03 and HEK293T cell lines are available in National Center for Biotechnology Information with the accession code SRP044809 [https://www.ncbi.nlm. nih.gov/sra/SRX661189[accn]] and [https://www.ncbi.nlm.nih.gov/sra/SRX665117 [accn]]. Details of the bat species used in this study can be found in [http://genomics. senescence.info/species/]. The source data underlying Figs. 1b, 2c-f, 5a-c, 6c, 6e, 6f, 7a, $7 \mathrm{c}$ and $7 \mathrm{~h}$ and Supplementary Figs. 2C-E are provided as a Source Data file. All data are available from authors upon request.

Received: 20 July 2017 Accepted: 14 May 2019

Published online: 27 June 2019

\section{References}

1. Cleeland, C. S. et al. Reducing the toxicity of cancer therapy: recognizing needs, taking action. Nat. Rev. Clin. Oncol. 9, 471-478 (2012).

2. Seluanov A., Gladyshev V. N., Vijg J., Gorbunova V. Mechanisms of cancer resistance in long-lived mammals. Nat. Rev. Cancer 18, 433-441 (2018).

3. Keane, M. et al. Insights into the evolution of longevity from the bowhead whale genome. Cell Rep. 10, 112-122 (2015).

4. Sulak M. et al. TP53 copy number expansion is associated with the evolution of increased body size and an enhanced DNA damage response in elephants. Elife 5, e11994 (2016).

5. Abegglen, L. M. et al. Potential mechanisms for cancer resistance in elephants and comparative cellular response to DNA damage in humans. J. Am. Med. Assoc. 314, 1850-1860 (2015).

6. Seluanov, A. et al. Hypersensitivity to contact inhibition provides a clue to cancer resistance of naked mole-rat. Proc. Natl Acad. Sci. USA 106, 19352-19357 (2009)

7. Tian, X. et al. High-molecular-mass hyaluronan mediates the cancer resistance of the naked mole rat. Nature 499, 346-349 (2013).

8. Gorbunova, V. et al. Cancer resistance in the blind mole rat is mediated by concerted necrotic cell death mechanism. Proc. Natl Acad. Sci. USA 109, 19392-19396 (2012) 
9. Nasser, N. J. et al. Alternatively spliced Spalax heparanase inhibits extracellular matrix degradation, tumor growth, and metastasis. Proc. Natl Acad. Sci. USA 106, 2253-2258 (2009).

10. Wang, L. F., Walker, P. J. \& Poon, L. L. M. Mass extinctions, biodiversity and mitochondrial function: are bats 'special'as reservoirs for emerging viruses? Curr. Opin. Virol. 1, 649-657 (2011).

11. In praise of bats. Nat. Ecol. Evol. 1, 0071 (2017) https://doi.org/10.1038/ s41559-017-0071.

12. Austad, S. N. Methusaleh's Zoo: how nature provides us with clues for extending human health span. J. Comp. Pathol. 142, S10-S21 (2010).

13. Austad, S. N. \& Fischer, K. E. Mammalian aging, metabolism, and ecology: evidence from the bats and marsupials. J. Gerontol. 46, B47-B53 (1991).

14. Wilkinson, G. S. \& South, J. M. Life history, ecology and longevity in bats. Aging Cell 1, 124-131 (2002).

15. Bradford, C., Jennings, R. \& Ramos-Vara, J. Gastrointestinal leiomyosarcoma in an Egyptian fruit bat (Rousettus aegyptiacus). J. Vet. Diagn. Invest. 22, 462-465 (2010).

16. McLelland, D. J., Dutton, C. J. \& Barker, I. K. Sarcomatoid carcinoma in the lung of an Egyptian fruit bat (Rousettus aegyptiacus). J. Vet. Diagn. Invest. 21, 160-163 (2009).

17. Siegal-Willott, J., Heard, D., Sliess, N., Naydan, D. \& Roberts, J. Microchipassociated leiomyosarcoma in an Egyptian fruit bat (Rousettus aegyptiacus). J. Zoo. Wildl. Med. 38, 352-356 (2007).

18. Zhang, G. et al. Comparative analysis of bat genomes provides insight into the evolution of flight and immunity. Science 339, 456-460 (2013).

19. Polo, S. E. \& Jackson, S. P. Dynamics of DNA damage response proteins at DNA breaks: a focus on protein modifications. Genes Dev. 25, 409-433 (2011).

20. Tubbs, A. \& Nussenzweig, A. Endogenous DNA damage as a source of genomic instability in cancer. Cell 168, 644-656 (2017).

21. Poirier, M. C. Linking DNA adduct formation and human cancer risk in chemical carcinogenesis. Environ. Mol. Mutagen. 57, 499-507 (2016).

22. Basu A. K. DNA Damage, Mutagenesis and cancer. Int. J. Mol. Sci. 19, E970 (2018).

23. Reisz, J. A., Bansal, N., Qian, J., Zhao, W. \& Furdui, C. M. Effects of ionizing radiation on biological molecules-mechanisms of damage and emerging methods of detection. Antioxid. Redox Signal. 21, 260-292 (2014).

24. Fernandez-Capetillo, O., Lee, A., Nussenzweig, M. \& Nussenzweig, A. H2AX: the histone guardian of the genome. DNA Repair 3, 959-967 (2004).

25. de Feraudy, S., Revet, I., Bezrookove, V., Feeney, L. \& Cleaver, J. E. A minority of foci or pan-nuclear apoptotic staining of $\gamma \mathrm{H} 2 \mathrm{AX}$ in the S phase after UV damage contain DNA double-strand breaks. Proc. Natl Acad. Sci. USA 107, 6870-6875 (2010).

26. Panier, S. \& Boulton, S. J. Double-strand break repair: 53BP1 comes into focus. Nat. Rev. Mol. Cell Biol. 15, 7-18 (2014).

27. Croco, E. et al. DNA damage detection by 53BP1: relationship to species longevity. J. Gerontol. A Biol. Sci. Med Sci. 72, 763-770 (2017).

28. Cortopassi, G. A. \& Wang, E. There is substantial agreement among interspecies estimates of DNA repair activity. Mech. Ageing Dev. 91, 211-218 (1996).

29. Montecucco, A., Zanetta, F. \& Biamonti, G. Molecular mechanisms of etoposide. EXCLI J. 14, 95-108 (2015).

30. Pang, B. et al. Drug-induced histone eviction from open chromatin contributes to the chemotherapeutic effects of doxorubicin. Nat. Commun. 4 , 1908 (2013).

31. de Lange, J. H. M. et al. Quantification by laser scan microscopy of intracellular doxorubicin distribution. Cytometry 13, 571-576 (1992).

32. Robey, R. W., Pluchino, K. M., Hall, M. D. \& Fojo, A. T. Revisiting the role of ABC transporters in multidrug-resistant cancer. Nat. Rev. Cancer 18, 452-464 (2018).

33. Wynne, J. W. et al. Proteomics informed by transcriptomics reveals Hendra virus sensitizes bat cells to TRAIL-mediated apoptosis. Genome Biol. 15, 532 (2014).

34. Kathawala, R. J., Gupta, P., Ashby, C. R. \& Chen, Z.-S. The modulation of $\mathrm{ABC}$ transporter-mediated multidrug resistance in cancer: a review of the past decade. Drug Resist. Updat. 18, 1-17 (2015).

35. Shi, Z. et al. Sildenafil reverses ABCB1- and ABCG2-mediated chemotherapeutic drug resistance. Cancer Res. 71, 3029-3041 (2011).

36. Loschmann, $\mathrm{N}$. et al. ABCB1 as predominant resistance mechanism in cells with acquired SNS-032 resistance. Oncotarget 7, 58051-58064 (2016).

37. Wolf, S. J. et al. An update on ABCB1 pharmacogenetics: insights from a 3D model into the location and evolutionary conservation of residues corresponding to SNPs associated with drug pharmacokinetics. Pharmacogenomics J. 11, 315-325 (2011).

38. Schinkel, A. H. et al. Normal viability and altered pharmacokinetics in mice lacking mdr1-type (drug-transporting) P-glycoproteins. Proc. Natl Acad. Sci. USA 94, 4028-4033 (1997).
39. Schinkel, A. H. et al. Disruption of the mouse mdrla P-glycoprotein gene leads to a deficiency in the blood-brain barrier and to increased sensitivity to drugs. Cell 77, 491-502 (1994).

40. Ueda, K., Cardarelli, C., Gottesman, M. M. \& Pastan, I. Expression of a fulllength cDNA for the human "MDR1" gene confers resistance to colchicine, doxorubicin, and vinblastine. Proc. Natl Acad. Sci. USA 84, 3004-3008 (1987).

41. Takeda, M. et al. The establishment of two paclitaxel-resistant prostate cancer cell lines and the mechanisms of paclitaxel resistance with two cell lines. Prostate 67, 955-967 (2007).

42. Pedrosa, P. et al. Combination of chemotherapy and Au-nanoparticle photothermy in the visible light to tackle doxorubicin resistance in cancer cells. Sci. Rep. 8, 11429 (2018)

43. Mizutani, T. et al. Genuine functions of P-glycoprotein (ABCB1). Curr. Drug Metab. 9, 167-174 (2008).

44. Stavrovskaya, A. A. \& Moiseeva, N. I. Non-canonical functions of the cellular transporter P-glycoprotein. Biochem. Moscow Suppl. Ser. A 10, 241-250 (2016).

45. Grandjean-Forestier F., Stenger C., Robert J., Verdier M., Ratinaud M. The PGlycoprotein 170: Just a Multidrug Resistance Protein or a Protean Molecule? (John Wiley and Sons, New York, 2009).

46. Zhu, H. et al. Role of MicroRNA miR-27a and miR-451 in the regulation of MDR1/P-glycoprotein expression in human cancer cells. Biochem. Pharm. 76, 582-588 (2008)

47. Chen, Z. et al. MiR-27a modulates the MDR1/P-glycoprotein expression by inhibiting FZD7/ $\beta$-catenin pathway in hepatocellular carcinoma cells. Cell Signal. 25, 2693-2701 (2013).

48. Yang, T. et al. MiR-223 modulates multidrug resistance via downregulation of ABCB1 in hepatocellular carcinoma cells. Exp. Biol. Med. 238, 1024-1032 (2013).

49. Hayashi, K. et al. HIV-Tat protein induces P-glycoprotein expression in brain microvascular endothelial cells. J. Neurochem. 93, 1231-1241 (2005).

50. Li, S. et al. LHBs can elevate the expression of MDR1 through HIF-1alpha in patients with $\mathrm{CHB}$ infection: a comparative proteomic study. Oncotarget 8, 4549-4562 (2017)

51. Leroy, E. M. et al. Fruit bats as reservoirs of Ebola virus. Nature 438, 575-576 (2005).

52. Li, W. et al. Bats Are Natural Reservoirs of SARS-Like Coronaviruses. Science 310, 676-679 (2005).

53. Kavanagh, J. N., Redmond, K. M., Schettino, G. \& Prise, K. M. DNA double strand break repair: a radiation perspective. Antioxid. Redox Signal. 18, 2458-2472 (2013)

54. Kakarougkas, A. \& Jeggo, P. A. DNA DSB repair pathway choice: an orchestrated handover mechanism. Br. J. Radiol. 87, 20130685 (2014).

55. Iyama, T. \& Wilson, D. M. 3rd DNA repair mechanisms in dividing and nondividing cells. DNA Repair 12, 620-636 (2013).

56. Podlutsky, A. et al. Comparative analysis of DNA repair pathways in mammals. FASEB J. 22, (1_supplement) 1239.32 (2008).

57. Yang, F., Teves, S. S. \& Kemp, C. J. Henikoff S. Doxorubicin, DNA torsion, and chromatin dynamics. Biochim Biophys. Acta Rev. Cancer 1845, 84-89 (2014).

58. Seim, I. et al. Genome analysis reveals insights into physiology and longevity of the Brandt's bat Myotis brandtii. Nat. Commun. 4, 2212 (2013).

59. Huang, Z., Jebb, D. \& Teeling, E. C. Blood miRNomes and transcriptomes reveal novel longevity mechanisms in the long-lived bat, Myotis myotis. BMC Genomics 17, 906 (2016).

60. Yong, K. S. M. et al. Bat-mouse bone marrow chimera: a novel animal model for dissecting the uniqueness of the bat immune system. Sci. Rep. 8, 4726 (2018).

61. Abolins, S. et al. The comparative immunology of wild and laboratory mice, Mus musculus domesticus. Nat. Commun. 8, 14811 (2017).

62. Harper, J. M., Salmon, A. B., Leiser, S. F., Galecki, A. T. \& Miller, R. A. Skin derived fibroblasts from long-lived species are resistant to some, but not all, lethal stresses and to the mitochondrial inhibitor rotenone. Aging Cell 6, 1-13 (2007).

63. Perlman, R. L. Mouse models of human disease: an evolutionary perspective. Evol. Med. Public Health 2016, 170-176 (2016).

64. Crameri, G. et al. Establishment, immortalisation and characterisation of pteropid bat cell lines. PLoS One 4, e8266 (2009).

65. Jones, T. R. et al. CellProfiler Analyst: data exploration and analysis software for complex image-based screens. BMC Bioinformatics 9, 482 (2008).

66. Olive, P. L. \& Banáth, J. P. The comet assay: a method to measure DNA damage in individual cells. Nat. Protoc. 1, 23-29 (2006).

67. Końca, K. et al. A cross-platform public domain PC image-analysis program for the comet assay. Mutat. Res. Genet. Toxicol. Environ. Mutagen. 534, 15-20 (2003). 


\section{Acknowledgements}

This study was supported by the grant, NRF2012NRF-CRP001-056 (to K.I. and L.F.W.) We thank Dr. Yanping Zhang for providing MEF cells. We thank Angela Andersen, Life Science Editors for editorial assistance. We thank Drs. Justin $\mathrm{Ng}$ and Aaron Irving for various reagents and helps. We also thank Drs. David L. Silver, Shang Li, Sudhakar Jha, Caroline Lee, Kazumitsu Ueda, Yasuhisa Kimura, Susan P.C. Cole, and Hiroaki Kato for all their advice and suggestions.

\section{Author contributions}

J.K., E.X.Y.S. and A.K.G. performed the experiments. I.H.M., D.L. and Y.T.C. conducted the bat capturing and tissue sample collections. J.K. and Y.T.C. established the primary cell lines and immortalised one of the cell lines. J.K., Y.I., L.F.W. and K.I. designed the experiments, analysed the results, and wrote the paper.

\section{Additional information}

Supplementary Information accompanies this paper at https://doi.org/10.1038/s41467019-10495-4.

Competing interests: The authors declare no competing interests.

Reprints and permission information is available online at http://npg.nature.com/ reprintsandpermissions/
Peer review information: Nature Communications thanks the anonymous reviewers for their contribution to the peer review of this work. Peer reviewer reports are available.

Publisher's note: Springer Nature remains neutral with regard to jurisdictional claims in published maps and institutional affiliations.

(c) (i) Open Access This article is licensed under a Creative Commons Attribution 4.0 International License, which permits use, sharing, adaptation, distribution and reproduction in any medium or format, as long as you give appropriate credit to the original author(s) and the source, provide a link to the Creative Commons license, and indicate if changes were made. The images or other third party material in this article are included in the article's Creative Commons license, unless indicated otherwise in a credit line to the material. If material is not included in the article's Creative Commons license and your intended use is not permitted by statutory regulation or exceeds the permitted use, you will need to obtain permission directly from the copyright holder. To view a copy of this license, visit http://creativecommons.org/ licenses/by/4.0/.

(C) The Author(s) 2019 